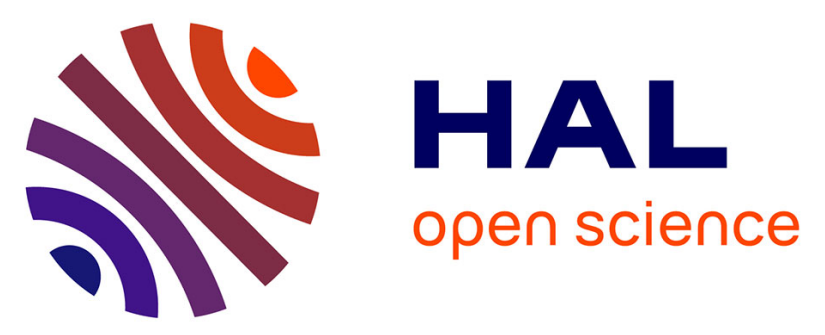

\title{
Overview of cobalt-mediated radical polymerization: Roots, state of the art and future prospects
}

\author{
Antoine Debuigne, Rinaldo Poli, Christine Jérôme, Jerome Robert, \\ Christophe Detrembleur
}

\section{- To cite this version:}

Antoine Debuigne, Rinaldo Poli, Christine Jérôme, Jerome Robert, Christophe Detrembleur. Overview of cobalt-mediated radical polymerization: Roots, state of the art and future prospects. Progress in Polymer Science, 2009, 34 (3), pp.211-239. 10.1016/j.progpolymsci.2008.11.003 . hal03184614

\section{HAL Id: hal-03184614 \\ https://hal.science/hal-03184614}

Submitted on 29 Mar 2021

HAL is a multi-disciplinary open access archive for the deposit and dissemination of scientific research documents, whether they are published or not. The documents may come from teaching and research institutions in France or abroad, or from public or private research centers.
L'archive ouverte pluridisciplinaire HAL, est destinée au dépôt et à la diffusion de documents scientifiques de niveau recherche, publiés ou non, émanant des établissements d'enseignement et de recherche français ou étrangers, des laboratoires publics ou privés. 


\section{Overview of Cobalt-Mediated Radical Polymerization :}

\section{Roots, State of the Art and Future Prospects.}

Antoine Debuigne, ${ }^{[\mathrm{a}]^{*}}$ Rinaldo Poli, ${ }^{[\mathrm{b}, \mathrm{c}]^{*}}$ Christine Jérôme, ${ }^{[\mathrm{a}]}$ Robert Jérôme, ${ }^{[\mathrm{a}]}$ Christophe Detrembleur $^{[\mathrm{a}]}$

${ }^{\text {[a] }}$ Center for Education and Research on Macromolecules, University of Liège (ULG), SartTilman, B6a, B-4000 Liège, Belgium.

${ }^{[b]}$ Laboratoire de Chimie de Coordination, UPR CNRS 8241 liée par convention à l’Université Paul Sabatier et à l'Institut National Polytechnique de Toulouse, 205 Route de Narbonne, 31077 Toulouse, France.

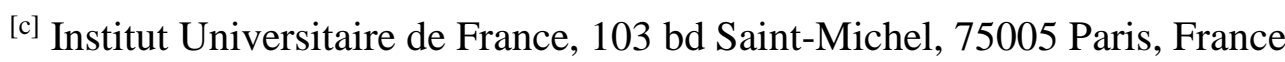

\footnotetext{
* to whom correspondence should be addressed - adebuigne@ulg.ac.be - fax 32-4-3663497
}

* to whom correspondence should be addressed - poli@lcc-toulouse.fr - fax 33-561553003 


\begin{abstract}
Controlled Radical Polymerization (CRP) techniques offer today a unique opportunity to properly design polymer chains and to adjust their chemical and physical properties. Among these techniques, cobalt-mediated radical polymerization (CMRP) distinguished itself by the high level of control imparted to the polymerization of acrylic and vinyl ester monomers, even for high molar masses. This article summarizes for the first time the advances in understanding and synthetic scope of CMRP since its discovery. Notably, the cobalt-carbon bond formation by dual contribution of reversible termination and degenerative chain transfer is discussed, as well as the impact of additives able to coordinate the metal. The potential of computational chemistry in the field of CMRP as a rationalization and predicting tool is also presented. These mechanistic considerations and achievements in macromolecular engineering will be discussed along with challenges and future prospects in order to assess the CMRP system as a whole.
\end{abstract}

\title{
Keywords
}

Controlled radical polymerization, Cobalt-mediated radical polymerization, copolymers, macromolecular engineering. 


\section{$\underline{\text { Table of Contents }}$}

1. Introduction

2. Roots of Cobalt-Mediated Radical Polymerization

2.1. Organocobalt complexes in carbon-carbon bond formation

2.2. Catalytic chain transfer polymerization (CCTP)

3. Cobalt-Mediated Radical Polymerization

3.1. General principles and preliminary considerations

3.2. Homopolymerization

3.2.1. Acrylic esters

3.2.2. Acrylic acid

3.2.3. Vinyl esters

3.2.3.1. Early results

3.2.3.2. Optimization

3.2.3.3. Mechanistic studies

3.2.4. Acrylonitrile

3.2.5. Conclusions

3.3. DFT calculations as a rationalizing and predicting tool

3.3.1. Calculations of $\mathrm{R}-\mathrm{Co}{ }^{\mathrm{III}} \mathrm{BDE}$

3.3.2. Calculations of $\mathrm{Co}^{\mathrm{II}}-\mathrm{L}$ and $\mathrm{Co}^{\mathrm{III}}-\mathrm{L}$ BDEs.

3.3.3. Perspectives for future work.

3.4. Improvement of the technology

3.5. Macromolecular Design

3.5.1. End-functionalization

3.5.2. Copolymerization

4. Concluding remarks and perspectives 
Acknowledgements

References 


\section{Introduction}

Polymers are essential in modern life. In order to meet the increasing demand for polymers with specific functionalities and applications, a range of synthetic methods has been developed. In this respect, Controlled Radical Polymerization (CRP) offers a unique opportunity for macromolecular engineering under mild experimental conditions. CRP has developed uninterruptedly [1] since its discovery in the mid-nineties [2]. Three methods rapidly established themselves initially: the nitroxide mediated radical polymerization (NMP) [3-6], the atom transfer radical polymerization (ATRP) [7-10], and the radical additionfragmentation chain transfer (RAFT) [11-14]. Subsequently, most efforts were devoted to the improvement of these systems and to their exploitation in macromolecular engineering. The choice of technique is mainly based on the adaptability of the controlling agents to an as wide as possible range of monomers, which is crucial for the design of complex polymeric architectures.

In the early nineties, at the very beginning of the CRP saga, the first controlled radical polymerizations using cobalt complexes as controlling species were reported by Wayland [15] and Harwood [16]. Despite the very high level of control of the acrylic monomers provided by this so-called cobalt-mediated radical polymerization (CMRP) system, the methodology long remained anecdotic because it appeared to be restricted to this type of monomers. Ten years later, the first example of CMRP of a non-acrylic monomer was reported by Jérôme et al.: bis(acetylacetonato)cobalt(II) proved high efficiency in controlling the radical polymerization of vinyl acetate (VAc) [17]. This breakthrough was all the more significant since VAc had long been a challenge for CRP because of the high reactivity of the propagating radical, resulting from the lack of stabilizing groups. For example, NMP is not suited for this monomer because the covalent bond between the nitroxide and the polymer 
chains is not sufficiently labile whatever the structure of the controlling agent [18]. ATRP in the presence of iron catalysts [19] and degenerative chain transfer polymerization based on alkyl iodides [20] offered poor and moderate control for VAc, respectively. Today, effective control of the VAc polymerization can also be achieved by reversible addition-fragmentation chain transfer (RAFT) using dithiocarbamates [21], macromolecular design via interchange of xanthates (MADIX) [21-30], organotellurium- and organostibine-mediated living radical polymerizations (TERP [31] and SBRP [32]).

The propensity of the cobalt-mediated radical polymerization to deal with such a challenging monomer for CRP was a strong incentive to pursue the development of organometallic-mediated radical polymerization (OMRP) [33], and particularly the one based on cobalt complexes. This review gives an overview of cobalt-mediated radical polymerization from its discovery to the most recent advances. Both mechanistic and macromolecular engineering aspects will be discussed in order to assess the system as a whole. Future prospects in CMRP, emphasized by this general presentation, will be discussed.

\section{Roots of Cobalt-Mediated Radical Polymerization}

In this part, we do not aspire to exhaustively overview the state of the art of cobalt chemistry prior the discovery of CMRP. We rather aim at pointing out important achievements in both organic and polymer chemistry involving cobalt, in order to deeply understand the CMRP principles as well as its possible side reactions.

\subsection{Organocobalt complexes in carbon-carbon bond formation}


Organocobalt(III) compounds have long attracted attention because of the facile homolytic cleavage of the cobalt-carbon bond under thermal or photolytic conditions [34-40]. Since the Co-C bond cleavage can be achieved under mild conditions, organocobalt complexes have been used as sources of carbon centered radicals for organic synthesis and in polymer chemistry.

First, it is essential to realize that the chemistry of cobalt complexes is closely related to the "Persistent Radical Effect" (PRE). This general concept, identified by Fisher et al. [41] in 1986 , rationalizes the highly specific formation of the cross-coupling product $\left(\mathrm{R}_{1}-\mathrm{R}_{2}\right)$ between a persistent radical $\left(\mathrm{R}_{1} \cdot\right)$ and a transient one $\left(\mathrm{R}_{2} \cdot\right)$ at the expense of the selftermination of the transient radicals $\left(\mathrm{R}_{2}-\mathrm{R}_{2}\right)$ [42]. At the early stage of the reaction, a tiny amount of the transient radical $\left(\mathrm{R}_{2} \cdot\right)$ disappears by self-termination, while the persistent radical $\left(\mathrm{R}_{1} \cdot\right)$ accumulates in the medium, favoring the formation of the cross-coupling product $\left(\mathrm{R}_{1}-\mathrm{R}_{2}\right)$. This concept notably explains the unusual stability of methylcobalamine $\mathbf{1}$ under photolytic treatment, the cobalt complex $\mathbf{2}$ and the methyl radical $\mathbf{3}$ being the persistent and the transient radicals, respectively (Fig. 1) [43]. In spite of the high propensity of methyl radicals $\mathbf{3}$ for cross-coupling reactions, ethane was not the major product. However, addition of carbon monoxide in the medium mainly resulted in the formation of acetylcobalamin $\mathbf{5}$ [43]. This experiment confirmed the "persistent radical" character of the $\mathrm{Co}^{\mathrm{II}}$ complex and illustrated the potential of alkyl-cobalt(III) as clean sources of radicals for radical addition reactions.

The thermal isomerization of the coenzyme B12 model is another perfect illustration of the PRE involving cobalt complexes, as reported by Finke and Daikh [44]. In this case, thermolysis of the benzyl-cobalt bond in the coenzyme B12 6 leads to an equilibrium between 6 and the benzyl migration product 7, with low formation of bibenzyl $(<0.001 \%)$, the radicalrecombination product (Fig. 2). 
The propensity of organocobalt complexes to display the persistent radical effect has largely been exploited in organic synthesis and more particularly for carbon-carbon bond formation, as assessed by an abundant scientific literature [45-47]. A typical example is the cobalt-mediated vinylation, described by Giese et al. [48] and illustrated in Fig. 3a. Upon photolysis, the glycosyl-cobalt complex 6 releases glycosyl radicals that add styrene. The coupling product of this radical adduct with the $\mathrm{Co}^{\mathrm{II}}$ complex was not isolated, but rather formed the alkene 7 by rapid dehydrocobaltation. This type of process was also studied by Branchaud et al. $[49,50]$ and Pattenden et al. [51] using alkyl- and acyl- cobalt complexes 8 and 10, as illustrated in Fig. 3b and 3c.

All these reactions follow the same general mechanism shown in Fig. 4. Exposition of the alkyl- or acylcobalt(III) complex 14 to light or heat triggers reversible homolytic Co-C bond cleavage and generates a carbon centered radical $\mathbf{1 5}$ and the persistent $\mathrm{Co}^{\mathrm{II}}$ complex. Subsequent addition of this carbon-centered radical to alkenyl compounds and reversible trapping of the newly formed radical $\mathbf{1 6}$ by the persistent $\mathrm{Co}^{\mathrm{II}}$ species to form $\mathbf{1 7}$ are followed by dehydrocobaltation with formation of the final unsaturated product $\mathbf{1 8}$.

This mechanism contains the basic reactions typical of cobalt complexes in a free radical polymerization medium. Indeed, just like small organic radicals, growing polymer radicals can react with cobalt complexes according to two pathways: reversible cobalt-carbon bond formation and dehydrocobaltation.

\subsection{Catalytic chain transfer polymerization (CCTP)}

The dehydrocobaltation observed in the cobalt-mediated vinylation is a key step in the catalytic chain transfer polymerization process (CCTP). As reported and discussed in details 
in several reviews [52-54], some cobalt(II) complexes, e.g. cobaloximes (e.g. complexes 13 and 19, in Fig. 5) and cobalt porphyrins (20) are effective chain transfer catalysts, responsible for moderating the molar mass of polymers prepared by radical polymerization, particularly poly(alkyl methacrylate)s (PMMAs).

According to the commonly accepted mechanism [52], a cobalt(II) complex reacts with a polymeric radical $\mathbf{2 1}$ forming an alkyl-cobalt(III) complex 22 or can undergo hydrogen atom abstraction by the cobalt with release of a polymer chain with an unsaturated end-group 23 and formation of a cobalt hydride 24. (Fig 6) The catalytic cycle is closed by generation of a primary radical 25 by hydrogen transfer from 24 to a methyl methacrylate (MMA) monomer unit, followed by propagation. Noteworthy, all growing radicals, including the primary radical 25, can be reversibly trapped by the cobalt(II) complex.

In this mechanism, the persistent radical effect is responsible for the reaction selectivity, i.e. the rapid cross-coupling reactions of the polymer radicals with the persistent Co ${ }^{\text {II }}$ complex vs. very limited self-termination of the polymer radicals. Moreover, all the polymer chains formed by CCTP bear an unsaturation at the $\omega$-chain end, which makes them potential macromonomers [55-60] and thus precursors of graft copolymers [61, 62]. For further information regarding the CCTP process, the reader is invited to read the review by Gridnev et al. [53, 54].

Vinylic monomers can be divided into two groups on the basis of their propensity to undergo catalytic chain transfer. Monomers having $\alpha$-methyl group with hydrogen atoms prone to abstraction, such as methacrylates, $\alpha$-methylstyrene and methacrylonitrile, are CCTactive monomers [52-54]. The second group includes monomers less prone to CCT reactions, such as acrylates, vinyl acetate, acrylonitrile, etc. In the total absence of hydrogen abstraction, the polymerization carried out in the presence of a cobalt complex is ruled in principle by the reversible Co-C bond cleavage and monomer addition, opening the way to cobalt-mediated 
controlled radical polymerization. In summary, depending on the propensity of the polymer to undergo hydrogen elimination and the ability of cobalt complex to promote H-abstraction, either "catalytic chain transfer polymerization" (CCTP) or "cobalt mediated radical polymerization" (CMRP) is favored, as emphasized in Fig. 6.

\section{Cobalt-Mediated Radical Polymerization}

\subsection{General principles and preliminary considerations}

A radical polymerization is considered to be under control when the termination reactions are negligible. This requires a controlling agent able to temporary deactivate the growing chains and minimize their instantaneous concentration. In the CMRP system, the deactivating species which caps the chains is the cobalt complex. Moreover, in order to limit the chain breaking reactions and obtain well-defined polymers, chain transfer reactions must also be negligible. An ideal CMRP process could thus be schematized according to Fig. 7.

Upon photolytic or thermal treatment, a preformed organocobalt(III) species undergoes Co-C homolytic cleavage with release of a persistent cobalt "radical" and a transient alkyl radical. In fact, the cobalt(II) product is not necessarily a simple radical, because its electronic configuration may involve in some cases three unpaired electrons (high spin configuration with a quartet ground state, vide infra), but always plays the role of a reversible radical trap just as a classical persistent radical. The reactive organic radical adds a first monomer unit and initiates the polymerization. In the absence of hydrogen atoms sensitive to abstraction on the growing polymer chains, and depending on the choice of the cobalt(II) species, the dehydrocobaltation pathway $\left(\mathrm{k}_{\mathrm{tr}}\right)$ could be disregarded at the expense of the temporary deactivation of the radical chains by the cobalt $\left(\mathrm{k}_{\mathrm{c}}\right)$. At this stage, the chains are 
considered as "dormant" and the radical species can be regenerated because of the low Co-C bond strength $\left(\mathrm{k}_{\mathrm{d}}\right)$. The polymer chains can thus further propagate $\left(\mathrm{k}_{\mathrm{p}}\right)$ until the next reversible deactivation. In other words, the CMRP process can be seen as a series of subsequent carbometallations of vinyl monomers. The instantaneous radical concentration must always be sufficiently low to avoid cross coupling reactions between the chains but sufficiently high to sustain polymerization. The Co-C strength, dictated by the choice of the cobalt complex structure for a given monomer, is thus a key parameter.

Among vinyl monomers, those deprived of $\alpha$-methyl groups, and particularly acrylic and vinyl ester monomers, are less prone to $\mathrm{H}$-abstraction by cobalt complexes. Consequently, these monomer families have been the first ones to be investigated by CMRP. In the subsequent sections of this review, the reactions are classified on the basis of the monomer. Within each section, the cobalt complexes are sorted by family, i.e. cobalt porhyrins and cobaloximes on one side and bis( $\beta$-diketonato)cobalt(II) derivatives on the other side.

\subsection{Homopolymerization}

\subsubsection{Acrylic esters}

In the early nineties, the groups of Harwood [16] and Wayland [15] discovered cobaltmediated radical polymerization almost simultaneously. Both were focused on the control of acrylate polymerization using cobaloxime and cobalt porphyrin complexes, respectively (see Tables 1 and 2).

As reported by Harwood et al. [16, 63-65] and patented a few years later [66, 67], (isopropylpyridinato)cobaloxime 27 (in Table 1), also known as organocobaloxime, is an 
efficient initiator and controlling agent for the radical polymerization of acrylates upon irradiation, as a result of the photolability of the cobalt-carbon bond. In a typical example, compound 27 photolytically initiated the polymerization of a $66 \%$ solution of ethyl acrylate in chloroform [65]. Under these conditions, the molar mass increased with the monomer conversion. However, a discrepancy between observed and expected molar masses appeared above a monomer conversion of $40 \%$ and the molar mass distribution was relatively high $\left(\mathrm{M}_{\mathrm{w}} / \mathrm{M}_{\mathrm{n}}>1.5\right)$. Interestingly, it was mentioned that the polydispersity tends to increase in the presence of a larger amount of chloroform (e.g., 50 volume percent) [65]. However, better results were obtained when the cobaloxime-mediated radical polymerization of acrylate was conducted in the presence of chloroform rather than in bulk or in other solvents (e.g., dimethylsulfoxide, benzene, dichloromethane). In these latter cases, it was suggested that chain transfer or termination competes with propagation because the polymer molar masses remained low and did not increase with conversion [65, 68]. It was proposed that the unique behavior of chloroform is due to its reaction with cobaloxime leading to chlorocobaloxime $[65,69,70]$ (eq. 1), which is inert under the polymerization conditions. Indeed, this would reduce the excess of cobaloxime in the polymerization medium and generate new radicals that can compensate radicals lost during the polymerization, allowing the polymerization to pursue in a pseudo-living manner. Although not perfect, a certain degree of control does exist and allows to synthesize poly(ethyl acrylate) with different molar masses by using various [monomer]/[organo-cobaloxime 27] molar ratios [65]. The possibility to control the acrylate polymerization in the presence of $\mathrm{Co}\left(\mathrm{dmgH}_{2}\right)_{2}, \mathbf{1 3}$ suggests a low contribution of the transfer reactions, contrary to the polymerization of methacrylates using the same complex [71].

$$
\mathrm{CHCl}_{3}+\mathrm{Co}\left(\mathrm{dmgH}_{2}\right)_{2} \mathrm{Py} \longrightarrow{ }^{\circ} \mathrm{CHCl}_{2}+\mathrm{ClCo}\left(\mathrm{dmgH}_{2}\right)_{2} \mathrm{Py}
$$


Interestingly, early work by Enikolopyan et al. reports the inhibition of acrylate polymerization when carried out in bulk at $60^{\circ} \mathrm{C}$ using azo-bis-isobutyronitrile (AIBN), as free radical initiator in the presence of cobalt porphyrin such as hematoporphyrin tetramethyl ether cobalt complex (Co(HPTME), 38 in Table 2) [72]. Later, other cobalt porphyrins were successfully used as mediators for efficient CMRP [15]. Tetra(mesityl)porphyrin organocobalt(III) complexes ((TMP) $\mathrm{Co}^{\mathrm{III}}-\mathrm{CH}_{2} \mathrm{C}\left(\mathrm{CH}_{3}\right)_{3} 31$ and (TMP)Co ${ }^{\mathrm{III}}-\mathrm{CH}\left(\mathrm{CO}_{2} \mathrm{CH}_{3}\right) \mathrm{CH}_{3}$ 32, see Table 2) were used as thermal-initiators for the methyl acrylate (MA) polymerization at $60^{\circ} \mathrm{C}$ in benzene solution $([\mathrm{MA}]=2.5 \mathrm{M})$ [15]. In this case, the alkyl radical $\left(\mathrm{R}^{\circ}\right)$ and the Co(TMP) complex 30, released by thermal homolysis of the Co-R bond, play the role of initiator and controlling agent, respectively. The linear molar mass increase with the monomer conversion and the relatively low polydispersity (1.1-1.3) indicated an effective controlled radical polymerization process. Interestingly, control was maintained above $70 \%$ monomer conversion even when molar masses beyond $200000 \mathrm{~g} / \mathrm{mol}$ were targeted. Moreover, the efficiency factor $\left(f=M_{n}\right.$ theor $/ M_{n}$ exp $)$ based on the ratio between the expected and the experimental molar masses was remarkably close to unity, which assessed the growth of one PMA chain per cobalt complex. The establishment of an equilibrium between the dormant organocobalt(III) species on one side and the growing radical and persistent Co ${ }^{\text {II }}$ (TMP) complex on the other side was the key step of this control. Self-termination of the growing radicals is effectively reduced according to the PRE. On the other hand, the methylene hydrogen abstraction by the metallic complex, leading to an unsaturated polyacrylate (CCTP), was minimized.

It was also demonstrated that the delicate balance between CCTP and CMRP for the polymerization of acrylate in the presence of cobalt complexes largely depends on the structure of the metallic complex, mainly on the steric demand of the ligands [73]. Typically, polymerizations initiated by organo-cobalt porphyrin complex 29 (in Table 2), parent of 
(tetraphenylporphyrinato)cobalt (Co(TTP), 28 in Table 2), yield an increased polydispersity (from 1.1 to 1.9 ) and stagnant molar masses as a function of monomer conversion, which is the clear signature of catalytic chain transfer (CCT) [73]. However, when Co(TTP) 28 was substituted for $\operatorname{Co}(\mathrm{TMP}) \mathbf{3 0}$, the steric hindrance of the tetramesitylporphyrin ligand prohibits the $H$-abstraction and allows the process to follow a controlled radical polymerization pathway [15]. Subsequently, Co(TTP) 28 definitely demonstrated its efficiency as catalytic chain transfer agent and its ability to provide macromonomers of PMMA by CCTP [74].

The (octabromotetramesityl)porphyrin organocobalt(II) complex ((Br8TMP)Co, 33 in Table 2) was also reported as efficient mediator for the acrylate polymerization at $60^{\circ} \mathrm{C}$ in benzene solution [75]. The control over the molecular parameters was comparable to that obtained with $\operatorname{Co}(\mathrm{TMP})$ 30, although the rate of polymerization was substantially higher. Indeed, the apparent first-order rate constant at $60^{\circ} \mathrm{C}\left(\mathrm{k}_{\mathrm{app}}\right)$ for the $\mathrm{MA}$ polymerization initiated by a preformed PMA-Co(Br8 $\mathrm{TMP})$ was 30 times larger than for PMA-Co(TMP). Substitution of bromine for hydrogen on the porphyrin core stabilizes cobalt in the oxidation state II and reduces the Co-C homolytic enthalpy, thereby shifting the equilibrium towards the active species and speeding up the polymerization. Since the organocobalt complex R$\mathrm{Co}\left(\mathrm{Br}_{8} \mathrm{TMP}\right)$ has a weaker $\mathrm{Co}-\mathrm{C}$ bond than $\mathrm{R}-\mathrm{Co}(\mathrm{TMP})$, it was possible to reduce the polymerization temperature. Indeed, $\left(\mathrm{Br}_{8} \mathrm{TMP}\right) \mathrm{Co}-\mathrm{CH}\left(\mathrm{CH}_{3}\right) \mathrm{OC}(\mathrm{O}) \mathrm{CH}_{3}$ (34 in Table 2) successfully initiated the controlled polymerization of methyl acrylate at room temperature [76].

In a more convenient approach, efficient initiators for CMRP were generated in situ by heating methyl acrylate at $60^{\circ} \mathrm{C}$ in benzene in the presence of a conventional free radical initiator (2,2'-azo-bis-(4-methoxy-2,4-dimethyl valeronitrile), V-70) and the cobalt(II) complex 30 [77]. 
The in-situ formation of the alkylcobalt(III) initiator is described as follows (Fig. 8). The tertiary carbon-centered radical formed by the thermal dissociation of the azo-initiator (eq. 2) is subjected to hydrogen abstraction by complex $\mathbf{3 0}$ leading to an unsaturated olefin and a cobalt hydride complex (eq. 3). This complex finally adds the monomer to form (TMP)Co ${ }^{\mathrm{III}}-\mathrm{CH}\left(\mathrm{CO}_{2} \mathrm{R}\right) \mathrm{CH}_{3}$, i.e., the actual initiator of methyl acrylate CMRP (eq. 4). The alkenes hydrocobaltation reaction was actually inspired from the reaction between the cobalt(II) porphyrin complex and AIBN, previously described by Wayland and co-workers $[78,79]$. Importantly, when the concentration of cobalt complex exceeded that of the radicals generated in the medium, the polymerization was extremely slow. However, production of a slight excess of radicals compared to cobalt $\left(\left[\mathrm{R}^{\circ}\right] /\left[(\mathrm{TMP}) \mathrm{Co}^{\mathrm{II}}\right]=1.2\right)$ led to a much faster polymerization. In this case, an induction period was observed prior to polymerization, corresponding to the time needed to inject an amount of radicals in the medium sufficient to convert all the $\mathrm{Co}^{\mathrm{II}}$ species into organocobalt(III) derivatives. The excess of radicals accelerates the polymerization without depriving the control of the polymerization. Moreover, the molar masses were close to the theoretical values corresponding to one polymer chain per cobalt porphyrin [77]. At that time, the CMRP was still described as a simple metallo-radical "stable free radical polymerization" (SFRP) governed by a combination-dissociation process, but this work announced a drastic change in the mechanistic interpretation of the CMRP system [77].

Indeed, in line with this work, it was notably demonstrated that the organocobalt porphyrin-mediated polymerization of acrylates can follow a reversible termination (RT) process but also a degenerative transfer (DT) pathway [80]. Such a dual behavior was previously demonstrated by mechanistic studies for organotellurium $[31,81]$ and organotitanium complexes [82, 83]. Unlike systems governed by RT, where the active growing polymer radicals are exclusively produced by homolytic dissociation of the bond 
between the polymer $(\mathrm{P})$ and the controlling agent $(\mathrm{X})$, a DT process requires a continuous influx of radicals, i.e. from a conventional radical initiator, that initiates tiny amounts of propagating chains during the whole polymerization process [84]. In a DT process, the active growing polymer chains $\left(\mathrm{P}^{\prime \circ}\right)$ are temporarily deactivated by reaction with the dormant species $(\mathrm{P}-\mathrm{X})$ with release of a new radical $\left(\mathrm{P}^{\circ}\right)$. Based on kinetics studies, it was demonstrated that the continuous injection of radicals in the CMRP polymerization medium turned the mechanism from a RT process (eq. 5), into a degenerative transfer process (eq. 6) $[80,85]$.

$$
\begin{aligned}
& \mathrm{P}-C o^{\mathrm{III}}+\mathrm{P}^{\circ}+\mathrm{Co}^{\mathrm{II}} \\
& \mathrm{P}-\mathrm{Co}^{\mathrm{III}}+\mathrm{P}^{\circ} \rightleftharpoons \mathrm{P}^{\prime}-\mathrm{Co}^{\mathrm{III}}+\mathrm{P}^{\circ}
\end{aligned}
$$

Such a phenomenon was first illustrated for the methyl acrylate polymerization conducted with (TMP)Co ${ }^{\mathrm{II}} 30$ in the presence and absence of an excess of $\mathrm{V}-70$ relative to the metal [80]. When a default of initiating radicals was used relative to (TMP)Co ${ }^{\mathrm{II}}$, the polymerization was very slow because of the low concentration of radicals involved in the equilibrium $\left(\mathrm{K}=\left[\mathrm{Co}^{\mathrm{II}}\right]\left[\mathrm{R}^{\circ}\right] /[\mathrm{Co}-\mathrm{R}]\right)$. In this case, the controlled character was ascribed to the PRE. However, when the total moles of radicals that enter the polymerization medium from V-70 exceeded the initial moles of (TMP)Co ${ }^{\mathrm{II}}$, a fast and controlled polymerization according to the DT mechanism was observed after the induction period. When a conventional V-70 initiator $\left(5.2 \times 10^{-4} \mathrm{M}\right)$ was added to a preformed CMRP initiator 32 [(TMP)Co$\left.\left.\mathrm{CH}\left(\mathrm{CO}_{2} \mathrm{CH}_{3}\right) \mathrm{CH}_{3}\right)\right]\left(1.0 \times 10^{-3} \mathrm{M}\right)$, the rate of the polymerization was close to the rate of an uncontrolled polymerization initiated by the same amount of V-70 without the cobalt complex, as expected for a DT process. Importantly, working under DT conditions allows reducing the concentration of the cobalt(II) complex in the medium and thus the probability 
for H-abstraction. Consequently, even the sterically less demanding tetra(pmethoxyphenyl)porphyrin cobalt(II) complex ((TAP)Co, 35), that normally promotes chain transfer reactions when used under RT conditions, successfully promotes the controlled radical polymerization of acrylates under DT conditions [80]. This change of mechanism is thus of prime importance for changing at demand the activity of the cobalt complex, and consequently, for increasing the selectivity of the desired reaction pathway.

\subsubsection{Acrylic acid}

Poly(acrylic acid) with low polydispersity and predetermined molar mass was prepared in water at $60^{\circ} \mathrm{C}$ by $\mathrm{CMRP}$ initiated by $\mathrm{V}-70$ in the presence of hydrosoluble tetra(3,5-disulfonatomesityl)porphinato cobalt complex (Co(TMPS), 36 in Table 2) [86, 87]. Similarly to acrylates, the radical polymerization of acrylic acid is mediated by both RT and DT pathways, depending on the amount of free radical initiator used relative to the cobalt complex. Typically, relatively well-defined $\left(\mathrm{M}_{\mathrm{w}} / \mathrm{M}_{\mathrm{n}} \sim 1.2-1.4\right)$ polyacrylic acid was obtained up to relatively high molar masses $\left(\mathrm{M}_{\mathrm{n}} \sim 300000 \mathrm{~g} / \mathrm{mol}\right)$, using a slight excess of $\mathrm{V}-70$ compare to the $\mathrm{Co}$ (TMPS) in water at $60^{\circ} \mathrm{C}$.

In parallel, polymerizations of methacrylic acid (MAA) initiated at $70^{\circ} \mathrm{C}$ by $4,4^{\prime}-$ azobis(4-cyanopenanoic acid) (V-501) in the presence of the less hindered tetra( $p$ sulfonatophenyl)porphyrin cobalt complex (Co(TSPP), 37 in Table 2) successfully led to macromonomers of poly(methacrylic acid) by CCTP [88].

\subsubsection{Vinyl esters}

\subsubsection{Early results}


In contrast to many vinyl monomers such as styrenes and (meth)acrylates, vinyl esters, particularly vinyl acetate, remained challenging monomers for controlled radical polymerization until recently. The main difficulty was the high reactivity of the propagating radicals due to the lack of stabilizing groups. However, many efforts have been devoted to the search for CRP systems able to deal with this monomer in order to exploit it in macromolecular engineering, since this monomer only polymerizes radically. Before the first example of CMRP of vinyl acetate was reported in 2005 [17], very few systems proved ability to mediate the radical polymerization of this monomer. Degenerative chain transfer using alkyl-iodides [20], RAFT using dithiocarbamates [21] and MADIX using xanthates [21-28] were the first reported methods that promoted a reasonable level of control for VAc. At that time, Jérôme's group developed the so-called "Quinone Transfer Radical Polymerization" (QTRP) [89-92], which proved efficient for controlling the radical polymerization of styrene when conducted in the presence of quinone derivatives associated with a metal catalyst. Acetylacetonato derivatives of nickel(II), manganese(II), aluminum(III) and particularly cobalt(II), were successfully used for QTRP of styrene [89-92]. With the hope of extending this method to other monomers, the QTRP of VAc was tested using phenanthrene quinone in the presence of bis(acetylacetonato)cobalt(II). In this case, a moderate control of the VAc polymerization was observed when the cobalt complex was used in stoichiometric amount compared to the quinone [93]. This result was rapidly and drastically improved when omitting the quinone in the same experiment. Indeed, it appeared that the bulk radical polymerization of VAc initiated at $30^{\circ} \mathrm{C}$ by $\mathrm{V}-70$ in the presence of $\mathrm{Co}(\mathrm{acac})_{2}(39$ in Table 3$)$ alone provided the best level of control achieved for this monomer until then [17]. This experiment encouraged us to cross the bridge between QTRP and CMRP and to further investigate the latter system. 


\subsubsection{Optimization}

In the initial procedure, the VAc polymerization was initiated in bulk at $30^{\circ} \mathrm{C}$ in the presence of $\mathrm{Co}(\mathrm{acac})_{2} 39$ using at least a threefold molar excess of initiator $(\mathrm{V}-70)$ relative to the controlling agent [17]. Under these conditions, an induction period of several hours was observed, which corresponds to the time required to consume all the $\mathrm{Co}^{\mathrm{II}}$ in the medium. As expected, this period was shorter when a higher $[\mathrm{V}-70] /[\mathrm{Co}]$ ratio was used, while preserving the control of the polymerization. It was subsequently demonstrated that one equivalent of V70 was sufficient, although the induction period was longer [94]. The ensuing VAc polymerization fulfilled all criteria of a controlled process, i.e. linear increase of the molar mass with the monomer conversion, molar mass distribution as low as 1.1-1.2, linear dependence of $\ln \left([\mathrm{M}]_{0} /[\mathrm{M}]\right)$ vs time. Moreover, there was very good agreement between experimental and expected molar masses considering one cobalt complex per chain. Furthermore, in contrast to the cobalt porphyrin used for the acrylate polymerization, $\mathrm{Co}(\mathrm{acac})_{2}$ is commercially available and inexpensive. By analogy to the CMRP based on cobalt porphyrin $[15,75,77]$ and cobaloxime [65], the mechanism proposed for the Co(acac) 2 mediated polymerization of VAc carried out in bulk was initially attributed to the reversible deactivation of the chains with the metal (Fig. 9). The equilibrium between the active growing PVAc chains and the dormant PVAc species end-capped by the Co complex could be possible by thermal homolytic cleavage of the metal-carbon bond [17]. However, the fact that the control of the CMRP process was not drastically affected by the large excess of initiator put the simple reversible termination model into question, as discussed hereafter.

Besides the temperature adjustment, which was optimum at $30^{\circ} \mathrm{C}$, the nature and amount of azo-initiator as well as the nature of the cobalt complex were investigated [95]. The reversible chain deactivation key step must be sensitive to the stereoelectronic properties of 
the cobalt complex. In addition to $\mathrm{Co}(\mathrm{acac})_{2}$, a series of commercially available cobalt derivatives were tested for the VAc polymerization initiated in bulk by V-70, such as sterically hindered bis(2,2',6,6'-tetramethylheptandionato)cobalt(II) (Co(TMHD) $)_{2}, 40$ in Table 3), but also the complexes bis(trifluoro-2,4-pentanedionato $)$ cobalt(II) $\left(\mathrm{Co}\left(\mathrm{F}_{3} \text {-acac }\right)_{2}, 41\right.$ in Table 3) and bis(hexafluoro-2,4-pentanedionato $)$ cobalt(II) $\left(\mathrm{Co}\left(\mathrm{F}_{6} \text {-acac }\right)_{2}, 42\right.$ in Table 3) containing electron-withdrawing $\mathrm{CF}_{3}$ substituents. $\left.\mathrm{Co}\left(\mathrm{F}_{3}-\mathrm{acac}\right)_{2}\right) \mathbf{4 1}$ behaved similarly to $\mathrm{Co}(\mathrm{acac})_{2} 39$ [95], yielding low polydispersity and good agreement between theoretical and experimental molar masses, whereas $\mathrm{Co}(\mathrm{TMHD})_{2} \mathbf{4 0}[93]$ and $\mathrm{Co}\left(\mathrm{F}_{6} \text {-acac }\right)_{2} 42$ [95] offered moderate and very poor control of the VAc polymerization, respectively. In the latter case, PVAc with high molar masses and broad polydispersity were formed without induction period, suggesting the inefficiency of this complex as trapping agent.

\subsubsection{Mechanistic studies}

Although the $\mathrm{Co}(\mathrm{acac})_{2}$ and cobalt porphyrin systems may appear similar, each has its own specificities. While the cobalt atom in the porphyrin system is constrained in a square planar conformation by the macrocyclic ligand and adopts a low spin $(S=1 / 2)$ configuration [96], the acetylacetonato system adopts a tetrahedral geometry and a high spin $(\mathrm{S}=3 / 2)$ configuration [97]. Both form mono and bis(ligand) adducts, which maintain the same spin state as the corresponding parent compound. The initiation mechanism also reflects the difference between these two systems. It was clearly established that the initiation of the VAc CMRP in the presence of $\mathrm{Co}(\mathrm{acac})_{2}$ does not consist in the in situ formation of cobalt hydride followed by hydrocobaltation of a first monomer unit, as was the case for cobalt porphyrin mediated acrylate polymerization represented in Fig. 8 [77]. Indeed, NMR analyses emphasized the presence of V-70 fragments at the $\alpha$-chain end of PVAc which confirmed that 
the VAc polymerization was actually initiated by radicals released by the thermolysis of V-70 (Fig. 10) [98]. This difference is most probably due to the lower propensity of $\mathrm{Co}(\mathrm{acac})_{2}$ for H-atom abstraction compared to Co porphyrins.

Since the contribution of a degenerative chain transfer mechanism was clearly evidenced for the cobalt porphyrin mediated radical polymerization of acrylate $[80,85]$, this possibility could not be ignored in the case of the $\mathrm{Co}(\mathrm{acac})_{2}$ mediated polymerization of VAc, especially when using excess V-70. As already mentioned above, the persistence of control in spite of the large excess of V-70 discredits the pure reversible-termination mechanism hypothesis [17, 98]. On the other hand, the observed successful resumption of VAc polymerization from a purified PVAc macroinitiator in the absence of additional V-70 is not compatible with a degenerative chain transfer mechanism [17]. This ambiguity was dispelled by Poli and Matyjaszewski [94]. On one hand, when isolated under strictly oxygen and moisture-free conditions, PVAc- $\mathrm{Co}(\mathrm{acac})_{2}$ failed to re-initiate the VAc polymerization, ruling out an RT process under these conditions. On the other hand, it was also demonstrated that addition of water to the purified macroinitiator made the chain extension possible. This clearly suggests that addition of water molecules favors the homolytic cleavage of the Co-C bond and activates the RT process. Water would exert this action by coordinating to the sixth coordinating site of cobalt, as will be analyzed more in detail below. This study emphasized for the first time the key role of Lewis base additives such as pyridine and water on the course of the VAc CMRP [94]. In summary, the CMRP mechanism of VAc in bulk in the presence of $\mathrm{Co}(\mathrm{acac})_{2}$ changes from degenerative chain transfer (DT) to reversible termination (RT) upon addition of water or pyridine, as supported by kinetic data [94].

The role of coordinating molecules on the CMRP was further investigated. Interestingly, when the VAc polymerization was initiated at $30^{\circ} \mathrm{C}$ with $\mathrm{V}-70$ in the presence of $\mathrm{Co}(\mathrm{acac})_{2}$ and 30 equivalents of pyridine, no induction period precedes the polymerization 
of VAc, contrary to the same experiment conducted without additive. In spite of the increase of the molar mass with the monomer conversion that assessed the controlled character of the polymerization, the experimental molar masses were more than twice higher than expected. The first observations is rationalized by the involvement of the cobalt complex in a ligand addition equilibrium, yielding the trans-octahedral $\mathrm{Co}(\mathrm{acac})_{2} \mathrm{Py}_{2}$ complex, as evidenced by NMR [94]. This equilibrium energetically stabilizes the Co ${ }^{\mathrm{II}}$ species, thereby shifting the Co ${ }^{\text {III }}$ PVAc bond dissociation equilibrium toward the formation of the active radical chain and opening access to the RT mechanism from the very beginning of the process. The second observation is a natural consequence of the slow production of the active $\mathrm{Co}^{\mathrm{III}}$-PVAc chains by the V-70 decomposition. The polymer $\mathrm{M}_{\mathrm{n}}$ becomes closer to the theoretical limit as more and more $\mathrm{Co}^{\mathrm{II}}$ is transformed into $\mathrm{Co}^{\mathrm{III}}$. The accordingly revised CMRP mechanism of the VAc is presented in Fig. 11.

In order to conduct an in-depth study of the CMRP mechanism, the synthesis of an alkylcobalt(III) model compound, able to initiate and mediate the polymerization, complex, was considered. Due to the high lability of the Co-C bond and sensitivity to oxygen, preparation and isolation of an alkylcobalt(III) complex supported by hard-donor ligands such as acetylacetonate (acac) was expected to be difficult. In fact, all envisioned strategies failed to provide the single $\mathrm{Co}$ adduct $\left(\mathrm{Co}(\mathrm{acac})_{2}-\mathrm{CH}(\mathrm{OAc}) \mathrm{CH}_{3}, \mathbf{4 3}\right.$ in Table 3) that mimics the PVAc dormant chains end-capped by Co(acac $)_{2}[99,100]$. However, reaction of V-70 with an excess of $\mathrm{Co}(\mathrm{acac})_{2}$ in the presence of $\mathrm{VAc}$ at $30^{\circ} \mathrm{C}$ gives access to low molecular weight cobalt(III) adducts $\left(\mathrm{Co}(\mathrm{acac})_{2}-\left(\mathrm{CH}(\mathrm{OAc}) \mathrm{CH}_{2}\right)_{<4}-\mathrm{R}_{0} ; \mathrm{R}_{0}=\mathrm{V}-70\right.$ fragment, 44 in Table 3), which consists of about three VAc units [99]. After isolation of $\mathbf{4 4}$ by chromatography, kinetic experiments were conducted using the alkylcobalt(III) adduct, free of V-70, as CMRP initiator for VAc in bulk at $30^{\circ} \mathrm{C}$. By using the $\mathrm{Co}(\mathrm{acac})_{2}-\left(\mathrm{CH}(\mathrm{OAc}) \mathrm{CH}_{2}\right)_{<4}-\mathrm{R}_{0} 44$ alone as initiator, a very slow polymerization was observed at $30^{\circ} \mathrm{C}$, which suggests that the PVAc- 
$\mathrm{Co}(\mathrm{acac})_{2}$ bond is relatively strong at this temperature. DFT calculations notably predicted that chelation by the carbonyl group of the last VAc unit provides an extra stabilization to the Co-C bond (see upper-left structure of $\mathbf{4 4}$ in Fig. 12 and the DFT section below). Then, addition of 1 equivalent of V-70 to the compound 44 in solution in VAc allows the polymerization to proceed mainly according to a DT process while maintaining a reasonable level of control (Route A in Fig. 12). However, in the presence of a small amount of water or pyridine (1-10 equivalents compare to the cobalt complex), the carbonyl group was replaced from the coordination sphere of $\mathbf{4 4}$ by the added Lewis base. In this case, the homolytic cleavage of the Co-C bond is facilitated, triggering the reversible termination mechanism (Route B in Fig. 12). Under these conditions, by adjusting the ratio between the monomer and 44, well-defined PVAc with molar masses as high as $150000 \mathrm{~g} / \mathrm{mol}$ and polydispersity as low as 1.1 was obtained, which is the best level of control ever observed for the radical polymerization of VAc by CMRP [99]. The role of coordinating molecules, which could also be the reaction solvent or the monomer itself, was crucial when the polymerization of other monomers and macromolecular engineering were envisioned by the CMRP technique (vide infra).

Very recently, the vinyl acetate polymerization was initiated by AIBN and/or V-70 at $60^{\circ} \mathrm{C}$ in the presence of (TMP)Co (30 in Table 2) [101] [102] [103] , which had previously been restricted to the control of the acrylic monomers. These experiments were carried out in deuterated benzene solutions in order to monitor the polymer yield by proton NMR. In spite of deviations from ideal CRP due to irreversible radical termination and transfer reactions, homopolymers with narrow molar mass distributions (1.1-1.3) were prepared at least at low monomer conversion (below 20\%). A kinetic study demonstrated the occurrence of a DT mechanism under these conditions. [103] 


\subsubsection{Acrylonitrile}

Well-defined poly(acrylonitrile) (PAN) containing materials have great potential, but the controlled polymerization of $\mathrm{AN}$ is challenging because of the high monomer reactivity and the low polymer solubility. Very recently, an in-depth study of the AN homopolymerization by CMRP was investigated using $\mathrm{Co}(\mathrm{acac})_{2}$ as controlling agent. The first attempts were carried out in dimethylformamide (DMF) solution at $30^{\circ} \mathrm{C}$ using $\mathrm{V}-70$ as initiator. However, the poor solubility of PAN in DMF at $30^{\circ} \mathrm{C}$ hampered the process [104]. In the better solvent dimethylsulfoxide (DMSO), a reasonable polymerization rate was observed (50\% conversion after $6 \mathrm{~h})$. Although the molar mass did not increase perfectly linearly with conversion, especially at the early stage of the experiment, some control was observed [104]. Indeed, PAN with relatively narrow molar mass distributions were obtained $\left(\mathrm{M}_{\mathrm{w}} / \mathrm{M}_{\mathrm{n}} \sim 1.4\right)$ and, for a given conversion, the molar mass increased when the $[\mathrm{AN}] /\left[\mathrm{Co}(\mathrm{acac})_{2}\right]$ ratio increased. However, the efficiency factor $(f)$ was much lower than unity (at 50\% monomer conversion $f=\mathrm{M}_{\mathrm{n}} \mathrm{th} / \mathrm{M}_{\mathrm{n} \exp }=0.3$ ).

On the other hand, when the AN polymerization was initiated at $0^{\circ} \mathrm{C}$ in DMSO with the alkylcobalt(III) compound 44 (Fig. 13), the AN polymerization fulfilled all criteria of a very well-controlled polymerization, i.e. the molar masses were close to the expected values (calculated considering the $[\mathrm{AN}] /[44]$ ratio), the polydispersity was close to 1.1 and the chain length increased linearly with the monomer conversion.[104]

Since compound $\mathbf{4 4}$ is free of azo-initiator residues, the polymerization of acrylonitrile in the presence of DMSO must follow a reversible termination RT mechanism. Coordination of the cobalt(II) complex by DMSO was confirmed by X-Ray analysis and supported by DFT calculations [104]. The latter technique also predicted the influence of such coordination on the Co-C bond dissociation enthalpy, as discussed in the DFT section below. This successful 
control of the acrylonitrile polymerization goes well beyond the extension of the CMRP system to another vinylic monomer. It also illustrates the extreme sensitivity of the CMRP system to the experimental conditions (solvent, temperature, initiating species, additives), opening many possibilities for the application to other monomers.

\subsubsection{Conclusions}

In general, the CMRP process has been successfully applied to four monomer families: acrylic esters, acrylic acid, vinyl esters and acrylonitrile. One characteristic of this process is that it can be conducted at low temperatures ranging from $0^{\circ} \mathrm{C}$ to $60^{\circ} \mathrm{C}$ depending on the Co-C bond strength. Moreover, it proved its efficiency on several occasions when high molar masses were targeted $(\mathrm{Mn}>200000 \mathrm{~g} / \mathrm{mol})$. However, although CMRP can manage different classes of monomers, it is striking that each of them is specifically controlled by one family of cobalt complexes. Indeed, cobalt porphyrin and cobaloximes are efficient to control the polymerization of acrylic monomers whereas bis( $\beta$-diketonato)cobalt(II) derivatives mainly deal with vinyl esters and acrylonitrile. For example, when conducted in the presence of $\mathrm{Co}(\mathrm{acac})_{2}$ the acrylate polymerization is fast and uncontrolled [95] due to a Co-C bond that is too labile as confirmed by DFT calculations [99]. As mentioned previously, one exception to this strict classification is the recent report on the CMRP of VAc at low monomer conversion using the tetra(mesityl porphyrinato)cobalt complex (30 in Table 2) [101-103].

A challenge in CMRP is to modulate the cobalt complex reactivity in order to deal with a large range of vinylic monomers with the same cobalt complex, which is of utmost importance when block copolymers and other macromolecular architectures are envisioned. In view of the recent results, this goal appears achievable by introduction of coordinating molecules in the polymerization medium. In this respect, computational calculations could be 
useful to predict the Co-C bond strength taking into account the structure of the monomer and the cobalt complex as well as the polymerization conditions (solvents, additives,...).

\subsection{DFT calculations as rationalizing and predicting tools}

The accuracy of theoretical calculations in terms of molecular structure, energetics and physical properties has made enormous progress in the last couple of decades. The application of computational tools, especially Density Functional Theory (DFT) methods, in view of their relatively good performance at comparatively low computational cost, is becoming routine in a variety of different areas of chemistry and controlled radical polymerization is no exception.

Calculations addressing CMRP have so far been limited to the $\mathrm{Co}(\mathrm{acac})_{2}$ system and to thermodynamic issues related to this polymerization technique. According to the accepted mechanism (e.g. see Fig. 7), the effective (pseudo-first order) polymerization rate constant is given by the expression of eq. 7 . The $\left(k_{\mathrm{d}} / k_{\mathrm{c}}\right)$ term is the equilibrium constant of the dormant species dissociation equilibrium. The precise nature of the $\mathrm{Co}^{\mathrm{III}}$ and $\mathrm{Co}^{\mathrm{II}}$ species involved depends on the presence in solution of donor ligands (L) that are capable of forming coordinative bonds with the two species and on the strength of these bonds, as shown in Fig. 14.

$$
k_{\text {eff }}=\left(k_{\mathrm{d}} / k_{\mathrm{c}}\right)\left(\left[\mathrm{R}-\mathrm{Co}^{\mathrm{III}}\right] /\left[\mathrm{Co}^{\mathrm{II}}\right]\right) k_{\mathrm{p}}
$$

$\mathrm{Co}^{\mathrm{II}}(\mathrm{acac})_{2}$ is known to adopt a tetrahedral geometry in dilute solution and the addition of neutral ligands may lead, if the $\mathrm{Co}^{\mathrm{II}}-\mathrm{L}$ bond is strong enough, to the formation of mono or (more frequently) bis-adducts, with respectively trigonal bipyramidal and trans-octahedral 
coordination geometries, as shown in Fig. 14. All these systems adopt a high spin electronic configuration (three unpaired electrons) because of the weak field of the acetylacetonato ligand. The $\mathrm{Co}^{\mathrm{II}}-\mathrm{L}$ bonds have a relatively low strength $\left[\right.$ small $\mathrm{BDE}_{1}\left(\mathrm{Co}^{\mathrm{II}}-\mathrm{L}\right)$ and $\mathrm{BDE}_{2}\left(\mathrm{Co}^{\mathrm{II}}-\right.$ $\mathrm{L})$ ] and are labile. The dormant complex $\mathrm{R}-\mathrm{Co}^{\mathrm{III}}(\mathrm{acac})_{2}$ may also bind $\mathrm{L}$, but no more than one molecule. Although simple alkylcobalt(III) complexes having these stoichiometries have not yet been isolated, [100] other alkylcobalt(III) compounds, mostly with porphyrin and corrin models (e.g. Schiff bases), are well known [105-108]. They can either adopt a square pyramidal configuration with an axial alkyl ligand, or a pseudo-octahedral geometry with trans $\mathrm{R}$ and L. Both systems are diamagnetic because of the strong ligand field of the alkyl ligand. The DFT calculations indicate that these geometries and the diamagnetic ground state are also adopted by the acac systems of $\mathrm{Co}^{\mathrm{III}}$.

The chain activation equilibrium will be more displaced toward the free radical and $\mathrm{Co}^{\mathrm{II}}$ (thereby speeding up the polymerization process) for weaker $\mathrm{R}-\mathrm{Co}^{\mathrm{III}}$ bonds and for ligands $\mathrm{L}$ that form stronger bonds with the resulting unsaturated $\mathrm{Co}^{\mathrm{II}}$ complex. Therefore, calculations have been focused on the evaluation of the strength of these bonds, namely the R$\mathrm{Co}^{\mathrm{III}}$ bond $\left[\mathrm{BDE}\left(\mathrm{R}-\mathrm{Co}^{\mathrm{III}}\right)\right.$ and $\left.\mathrm{BDE}\left(\mathrm{R}-\mathrm{Co}_{\mathrm{L}}{ }^{\mathrm{III}}\right)\right]$, the $\mathrm{Co}^{\mathrm{III}}-\mathrm{L}$ bond $\left[\mathrm{BDE}\left(\mathrm{Co}^{\mathrm{III}}-\mathrm{L}\right)\right]$, and the $\mathrm{Co}^{\mathrm{II}}-$

$\mathrm{L}$ bonds $\left[\mathrm{BDE}_{1}\left(\mathrm{Co}^{\mathrm{II}}-\mathrm{L}\right)\right.$ and $\left.\mathrm{BDE}_{2}\left(\mathrm{Co}^{\mathrm{II}}-\mathrm{L}\right)\right]$. The influence on these bonds by the presence/absence and chemical nature of $\mathrm{L}$ is of particular interest.

\subsubsection{Calculations of $R-C o^{I I I} B D E$}

Previous calculations of $\mathrm{Co}^{\mathrm{III}}$-alkyl bond strengths had mostly been confined to the vitamin B12 system [109-113]. There is a major difference between the porphyrin and corrin model systems and $\mathrm{Co}(\mathrm{acac})_{2}$ : the stronger field of the porphyrin and Schiff base ligands forces $\mathrm{Co}^{\mathrm{II}}$ in the low spin configuration $(\mathrm{S}=1 / 2)$. Therefore, the R-Co ${ }^{\mathrm{III}}$ homolytic bond 
rupture leaves a single unpaired electron on both the radical and the cobalt atom when the latter is coordinated by the stronger porphyrin or Schiff base ligands, whereas the same process is accompanied by the unpairing of two additional electron from a metal $d$ orbital for the $\mathrm{Co}(\mathrm{acac})_{2}$ system, see Fig. 15. This is because the energetic separation between filled and empty orbitals is smaller in the presence of the low-field acetylacetonato ligands.

When addressing the specific question of the Co-C bond strength in (acac) ${ }_{2} \mathrm{Co}^{\mathrm{III}}-\mathrm{R}$ systems, a major computational problem concerns the change of spin state associated to the homolytic rupture (from spin singlet $(\mathrm{S}=0)$ for $\mathrm{Co}^{\mathrm{III}}-\mathrm{R}$ to a global spin triplet $(\mathrm{S}=1)$ resulting from the combination of the quartet $(\mathrm{S}=3 / 2)$ of the $\mathrm{Co}^{\mathrm{II}}$ complexes and the doublet $(\mathrm{S}=1 / 2)$ of the free organyl radical). For processes where a change of spin is involved, DFT methods provide results that are wildly different, depending on the type of functional used. Accurate bond enthalpies are accessible in principle through high level computational methods, but this is quite impractical for molecules with the size of those of interest here. A detailed discussion of this phenomenon is available in other specialized articles [114-117]. In this review, we limit ourselves to mentioning that the most typical and frequently used "hybrid" functionals such as B3LYP are known to overestimate the relative stability of higher spin states, whereas "pure" functionals such as BP86 often do the reverse. Unfortunately, no "rule of thumb" is available for the selection of the best functional for a specific problem. An appropriate choice would be made possible by comparing calculated and experimentally available parameters, or by comparison with much higher level ab initio calculations, believed to provide a closer approximation of the experimental value. Modified hybrid functionals with a reduced admixture of the exact exchange have been developed using these approaches on simple and well investigated systems, for instance the $\mathrm{CO}$ dissociation from $\mathrm{Fe}(\mathrm{CO})_{5}$ (going from singlet to triplet) [115]. For the typical systems used in CMRP, the first approach is impossible because Co-R BDEs are not experimentally available, while the second one is prohibitive 
given the relative complexity of the systems. Thus, it is wise to compare the results obtained with different functionals and to focus the analysis on trends rather than on absolute values.

Calculations of $\mathrm{BDE}\left(\mathrm{R}-\mathrm{Co}^{\mathrm{III}}\right)$ and $\mathrm{BDE}\left(\mathrm{R}-\mathrm{Co}_{\mathrm{L}}{ }^{\mathrm{III}}\right)$ values (see Fig. 14), carried out with the B3LYP functional, were initially restricted to a model system where $\mathrm{R}=\mathrm{CH}_{3}$ [94] and later extended to $\mathrm{R}=\mathrm{CH}\left(\mathrm{OCH}_{3}\right) \mathrm{CH}_{3}$ (as a model of poly(vinyl ether) (PVE)), $\mathrm{CH}\left(\mathrm{OOCCH}_{3}\right) \mathrm{CH}_{3}$ (as a model of $\left.\mathrm{PVAc}\right), \mathrm{CH}(\mathrm{CN}) \mathrm{CH}_{3}$ (as a model of PAN), $\mathrm{CH}\left(\mathrm{CO}_{2} \mathrm{CH}_{3}\right) \mathrm{CH}_{3}$ (as a model of PMA), and $\mathrm{CH}\left(\mathrm{CH}_{3}\right)_{2} \mathrm{CN}$ (primary radical generated from AIBN) $[99,104]$. These results (see Table 4) confirm the expected dependence of the bond strength on radical stabilization.

The calculations on the model $\mathrm{CH}_{3}-\mathrm{Co}(\mathrm{acac})_{2}(\mathrm{~L})$ system with $\mathrm{L}=\mathrm{py}, \mathrm{NMe}_{3}, \mathrm{NH}_{3}$ and $\mathrm{H}_{2} \mathrm{O}$ show that the $\mathrm{L}$ coordination strengthens the $\mathrm{Co}-\mathrm{CH}_{3}$ bond by $3-4 \mathrm{kcal} \mathrm{mol}^{-1}$, with apparently little influence of the nature of L [94]. This strengthening phenomenon can be associated to an energetically more favorable relaxation of $\mathrm{Co}(\mathrm{acac})_{2}$ from square planar to tetrahedral than $\mathrm{Co}(\mathrm{acac})_{2}(\mathrm{~L})$ from square pyramidal to trigonal bipyramidal. The practical result is a negative effect on the radical activation process. It is portrayed qualitatively in Fig. $14\left[\mathrm{BDE}\left(\mathrm{R}-\mathrm{CoL}_{\mathrm{L}}^{\mathrm{III}}\right)>\mathrm{BDE}\left(\mathrm{R}-\mathrm{Co}^{\mathrm{III}}\right)\right]$. However, it is outweighed by the positive effect of further L coordination to yield 6-coordinate $\mathrm{Co}(\mathrm{acac})_{2}(\mathrm{~L})_{2}$, which energetically stabilizes the $\mathrm{Co}^{\mathrm{II}}$ complex thereby shifting the radical activation equilibrium, vide infra. Analogous calculations on $\mathrm{R}-\mathrm{Co}(\mathrm{acac})_{2}(\mathrm{~L})$ with $\mathrm{R}=\mathrm{CH}\left(\mathrm{OOCH}_{3}\right) \mathrm{CH}_{3}$ and $\mathrm{CH}(\mathrm{CN}) \mathrm{CH}_{3}$ and with $\mathrm{L}=$ AN, DMF and DMSO also show an effect of ligand coordination, but this is now modulated by the steric effects: the smaller AN ligand shows a strengthening effect, the slightly more encumbering DMF ligand has a smaller strengthening effect, and the bulkier DMSO ligand has essentially no effect (strengthening by only $1.6 \mathrm{kcal} \mathrm{mol}^{-1}$ for $\mathrm{CH}(\mathrm{CN}) \mathrm{CH}_{3}$; weakening by $1.2 \mathrm{kcal} \mathrm{mol}^{-1}$ for $\left.\mathrm{CH}\left(\mathrm{OOCCH}_{3}\right) \mathrm{CH}_{3}\right)$ [104]. These findings are very much in line with 
previous work on the vitamin B12 models, where the steric bulk of the axial ligand was shown to have an important effect on the Co ${ }^{\mathrm{III}}-\mathrm{R}$ labilization $[37,118]$.

The PVAc model system, $\mathrm{CH}\left(\mathrm{OOCCH}_{3}\right) \mathrm{CH}_{3}$, deserves special consideration, because the acetate function of the Co-capped monomer unit is suitably placed to saturate the coordination sphere of $\mathrm{Co}^{\mathrm{III}}$ by chelation. Thus, the dormant form of the Co-capped PVAc growing chain, PVAc-Co(acac)2, is not 5-coordinated but rather 6-coordinated, as already illustrated above in Fig. 12, even in the absence of external donor ligands. This chelation provides an extra $6 \mathrm{kcal} \mathrm{mol}^{-1}$ of stability to the alkylcobalt(III) system, at the B3LYP level, and competes effectively with the coordination of the less strongly bonded L ligands, see below [99].

As stated above, the nature of the functional used in the calculation has an important effect on processes where a change of spin state occurs. Use of the B3PW91* functional [115], where the contribution of exact exchange is reduced relative to B3LYP, has the effect of increasing the calculated R-Co ${ }^{\mathrm{III}} \mathrm{BDE}$ by ca. $6 \mathrm{kcal} \mathrm{mol}^{-1}$ on average (see Table 4), but the relative trend of bond strengths as a function of $\mathrm{R}$ remains unaltered.

\subsubsection{Calculations of Co ${ }^{I I}-L$ and Co ${ }^{I I I}-L$ BDEs.}

The Co-L bond strengths studied so far are listed in Table 5. One point worthy of note is that for those bonds that have been investigated at both B3LYP and B3PW91* levels, the values obtained do not differ too much $\left(2 \mathrm{kcal} \mathrm{mol}^{-1}\right.$ at the most $)$ between the two functionals. This is because the ligand binding does not result in a change of spin state ( $\mathrm{S}=0$ for the $\mathrm{Co}^{\mathrm{III}}$ systems, $3 / 2$ for the $\mathrm{Co}^{\mathrm{II}}$ systems). 
The calculations of $\mathrm{BDE}_{1}\left(\mathrm{Co}^{\mathrm{II}}-\mathrm{L}\right)$ and $\mathrm{BDE}_{2}\left(\mathrm{Co}^{\mathrm{II}}-\mathrm{L}\right)$ confirms the experimentally established weakness of these bonds. The $\mathrm{BDE}\left(\mathrm{Co}^{\mathrm{III}}-\mathrm{L}\right)$ values are slightly greater (for instance, $c f$. the values of $\mathrm{BDE}\left(\mathrm{Co}^{\mathrm{III}}-\mathrm{L}\right)$ and $\mathrm{BDE}_{1}\left(\mathrm{Co}^{\mathrm{II}}-\mathrm{L}\right)$ for $\mathrm{L}=\mathrm{NMe}_{3}$ and $\mathrm{H}_{2} \mathrm{O}\left(\mathrm{R}=\mathrm{CH}_{3}\right)$ and for $\mathrm{L}=\mathrm{AN}\left(\mathrm{R}=\mathrm{CH}\left(\mathrm{OOCCH}_{3}\right) \mathrm{CH}_{3}\right.$ or $\left.\mathrm{CH}(\mathrm{CN}) \mathrm{CH}_{3}\right)$. As shown in Fig. 14, the values of $\mathrm{BDE}\left(\mathrm{Co}^{\mathrm{III}}-\mathrm{L}\right), \mathrm{BDE}_{1}\left(\mathrm{Co}^{\mathrm{II}}-\mathrm{L}\right), \mathrm{BDE}\left(\mathrm{R}_{-} \mathrm{Co}^{\mathrm{III}}\right)$ and $\mathrm{BDE}\left(\mathrm{R}-\mathrm{Co}_{\mathrm{L}}{ }^{\mathrm{III}}\right)$ are tied together in a Hess cycle. Thus, the stronger bond of $\mathrm{L}$ with $\mathrm{Co}^{\mathrm{III}}$ than with $\mathrm{Co}^{\mathrm{II}}\left(\mathrm{BDE}_{1}\left(\mathrm{Co}^{\mathrm{II}}-\mathrm{L}\right)\right)$ is related to the fact that the R-Co ${ }^{\mathrm{III}}$ bond is stronger in the 6-coordinate $\mathrm{R}-\mathrm{Co}(\mathrm{acac})_{2}(\mathrm{~L})$ than in the 5coordinate $\mathrm{R}-\mathrm{Co}(\mathrm{acac})_{2}$, at least for sterically unencumbering L molecules.

The calculations show that $\mathrm{BDE}_{1}\left(\mathrm{Co}^{\mathrm{II}}-\mathrm{L}\right)$ is smaller than $\mathrm{BDE}_{2}\left(\mathrm{Co}^{\mathrm{II}}-\mathrm{L}\right)$ for the unencumbering and poorly coordinating AN ligand, about the same for ligands of intermediate size $\left(\mathrm{NMe}_{3}, \mathrm{py}, \mathrm{DMF}\right)$ and greater for the bulkier and more strongly binding DMSO ligand. Two trends emerge from these calculations: electronic factors favor ligand binding in the order $\mathrm{Co}(\mathrm{acac})_{2}(\mathrm{~L})>\mathrm{Co}(\mathrm{acac})_{2}$, whereas steric factors show the opposite trend.

The specific use of the calculated $\mathrm{R}-\mathrm{Co}^{\mathrm{III}}$ and $\mathrm{Co}^{\mathrm{II} / \mathrm{III}}-\mathrm{L}$ bond strength rationalizes rather well the better ability of $\mathrm{Co}(\mathrm{acac})_{2}$ to control the polymerization of $\mathrm{AN}$ in the coordinating solvent DMSO, relative to non coordinating solvents (toluene or anisole). As shown in Fig. 16, the dormant chain in a non coordinating solvent is likely to be the 5coordinated (acac) $)_{2} \mathrm{Co}-\mathrm{PAN}$ (modeled in the calculation by $(\mathrm{acac})_{2} \mathrm{Co}-\mathrm{CH}(\mathrm{CN}) \mathrm{CH}_{3}$ ), since coordination of AN to this species provides only very small stabilization, probably insufficient to counter the negative effect of the entropy term. Radical dissociation yields $\mathrm{Co}(\mathrm{acac})_{2}$, which also does not enjoy stabilization by AN coordination (both 5-coordinate $\mathrm{Co}(\mathrm{acac})_{2}(\mathrm{AN})$ and 6-coordinate $\mathrm{Co}(\mathrm{acac})_{2}(\mathrm{AN})_{2}$ have higher enthalpy and therefore even higher free energy). The radical trapping process provides $6.7 \mathrm{kcal} \mathrm{mol}^{-1}$ of enthalpic stabilization but is disfavored entropically. In the presence of DMSO, both the $\mathrm{Co}^{\mathrm{III}}$ and $\mathrm{Co}^{\mathrm{II}}$ complexes become coordinatively saturated, to yield $\mathrm{PAN}-\mathrm{Co}{ }^{\mathrm{III}}(\mathrm{acac})_{2}(\mathrm{DMSO})$ and 
$\mathrm{Co}^{\mathrm{II}}(\mathrm{acac})_{2}(\mathrm{DMSO})_{2}$, respectively. In fact, the latter complex was isolated in crystalline form and characterized by X-ray diffraction. Thus, the trapping process involves loss of DMSO from $\mathrm{Co}^{\mathrm{II}}(\mathrm{acac})_{2}(\mathrm{DMSO})_{2}$ followed by radical addition. The enthalpic stabilization is $6.6 \mathrm{kcal}$ mol $^{-1}$, very similar to that obtained in the absence of DMSO, but the process is now essentially isoentropic. The rationalization of the solvent effect on the initiation of AN polymerization starting from a PVAc-Co(acac $)_{2}$ macroinitiator will be addressed below within the block copolymer section.

\subsubsection{Perspectives for future work.}

The kinetic aspects of CMRP has not yet been addressed. Although the rate of polymerization (eq. 7) depends only on the thermodynamics (equilibrium position) of the organocobalt complex formation process, the trapping rate is of paramount importance for insuring low polydispersities. A trapping process having higher activation barriers will result in a lower trapping efficiency and thus in a greater accumulation of the active radical concentration, with negative effects on terminations and polydispersity. Understanding how the nature of the ligand and the spin state change affect this trapping barrier would be particularly useful for the optimization of the reversible deactivation process. For instance, as shown schematically in Fig. 17, path (a) yields a lower equilibrium concentration of active radical and a faster trapping rate, whereas path (b) would yield a greater concentration of radicals and a less efficient trapping process. Both paths (a) and (b) refer to adiabatic processes without a change of total spin (e.g. when the Co complex has $S=1 / 2$ ). Path (c) illustrates the case of a change of spin, as in the case of the $\mathrm{Co}(\mathrm{acac})_{2}$ system. In such case, the explicit calculation of the Minimum Energy Crossing Point (MECP) [119, 120] would be necessary to evaluate the activation barrier. Calculations on associative exchange pathways, 
as shown in Fig. 18, would also be necessary in order to evaluate the efficiency of systems where the degenerative transfer mechanism predominates. For instance, a system yielding pathway (d) would undergo a slower exchange, and therefore poorer control, than a system yielding pathway (e).

Finally, a point which is still totally obscure is the role of the metal coordination sphere in determining the aptitude of the complex to yield catalytic chain transfer versus reversible deactivation. This is again a kinetic issue, determined by the relative height of two activation barriers: that for radical trapping to form an organometallic dormant chain versus that for $\mathrm{H}$ atom transfer to form the hydride complex and a dead chain with an unsaturated chain end. A greater degree of understanding of which parameters regulate these two barriers will give guidance for the development of tailored complexes with specific roles. In our opinion, the computational tool can also help in this direction.

\subsection{Improvement of the technology}

In order to bring the CMRP process closer to industrial standards, several questions need to be addressed, i.e. compatibility of the technique with aqueous dispersed media, use of widespread initiators, recovery and recycling of the cobalt complex. The $\mathrm{Co}(\mathrm{acac})_{2} 39$ mediated radical polymerization of VAc was used as model to address these challenges.

Most of the radical polymerization processes are carried out in aqueous dispersed media because of the easy control of heat transfer, absence of organic solvent and higher monomer conversion. Therefore, the CMRP of VAc was tentatively carried out in aqueous dispersed media, such as suspension [98, 121] and miniemulsion [98, 122], rather than in bulk. In this approach, several questions appeared obvious such as the much higher affinity of the controlling agent $\left(\mathrm{Co}(\mathrm{acac})_{2}\right)$ for the water phase compared to the monomer and the effect 
of a large amount of water available to coordinate the cobalt complex. The first problem was tackled by using a preformed PVAc macroinitiator end-capped by cobalt, which is sufficiently hydrophobic to locate the cobalt in the monomer phase. Typically, the PVAc-Co(acac $)_{2}$ macroinitiator was dissolved in VAc prior to dispersion in water. Poly(vinyl alcohol) (PVOH, 0.16 wt $\%$ in water) and sodium dodecyl sulfate (SDS, 0.9 wt \% in water) were used as surfactant for the suspension and the miniemulsion procedures, respectively. In the latter case, ultrasonication was necessary to obtain the nanometric droplets, precursors of the latex particles. The polymerizations, carried out at $30^{\circ} \mathrm{C}$, always followed a controlled pathway even at high monomer conversion (above 75\%) and relatively high molar masses ( 100000 $\mathrm{g} / \mathrm{mol}$ ). However, the molar masses were higher than predicted. Interestingly, compare to the bulk, the polymerization rate was high to extremely high for the suspension $(75 \%$ conversion in $70 \mathrm{~min}$ ) and the miniemulsion (90\% conversion in $30 \mathrm{~min}$ ), respectively. Although the water content in the monomer droplet might coordinate cobalt and participate to speeding up the reaction $[94,99]$, such a kinetic effect was demonstrated to be mainly due to a partitioning effect, i.e. the continuous departure of the deactivating species $\left(\mathrm{Co}(\mathrm{acac})_{2}\right)$ into the water phase (Fig. 19) [99, 121]. At the end, micrometric beads and latexes of well-defined PVAc were obtained in suspension and miniemulsion, respectively.

Throughout our work, the use of V-70 as initiator for the CMRP has been chosen because of its low decomposition temperature $\left(\mathrm{t}_{1 / 2}=12 \mathrm{~h}\right.$ at $\left.30^{\circ} \mathrm{C}\right)$, which is compatible with the optimum temperature $\left(30^{\circ} \mathrm{C}\right)$ dictated by the thermal lability of the Co-C bond. Within the search for a substitute for V-70, redox initiating systems appeared as an interesting alternative because they are effective at room temperature and largely used at the industrial scale [123]. Therefore, the CMRP of VAc was initiated at $30^{\circ} \mathrm{C}$ from a mixture of peroxide (lauroyl peroxide or benzoyl peroxide) as oxidant, ascorbic acid as reducing agent and $\mathrm{Co}(\mathrm{acac})_{2} 39$ with the dual role of reducing and controlling agent. Under these conditions, the 
polymerization was fast and remained controlled. However, the initiator efficiency ( $\mathrm{f}=$ $\left.\mathrm{M}_{\mathrm{n}, \mathrm{th}} / \mathrm{Mn}_{\text {,exp }}\right)$ was low $(0.1 \leq \mathrm{f} \leq 0.25)$, suggesting that $\mathrm{Co}(\mathrm{acac})_{2}$ also participated to the redox initiation. When ascorbic acid was replaced by citric acid, the polymerization was much faster while maintaining good control. Substitution of redox systems for V-70 underlined the sensitivity of the Co complex towards oxidizing agents but also emphasized the potential for a reaction scale-up [123].

Except when the metal content in the polymer falls down into the ppm range, it becomes essential to address the question relative to its elimination and recovery. Since a stoichiometric amount of cobalt compared to the polymer chains is intrinsic to the CMRP mechanism, it was of utmost importance to develop an efficient method for polymer purification and for metal complex recycling. In this respect, thermal treatment of the PVAc$\mathrm{Co}(\mathrm{acac})_{2}$ chains with a nitroxide or a thiol at the end of the polymerization was shown to drastically reduce the cobalt content in the polymer matrix [124]. After homolytic Co-C bond cleavage, the released PVAc radical was scavenged by the nitroxide or abstracted the thiol $\mathrm{H}$ atom, respectively. However, although a simple filtration through a silica column allows the removal of the metal from the polymer matrix, these approaches are not compatible with the recovery and recycling of the cobalt complex. In order to solve this problem, the cobalt complex was supported on the surface of inert materials [125]. Acetylacetone groups were first grafted onto silica or Merrifield resin, followed by a ligand exchange reaction involving $\mathrm{Co}(\mathrm{acac})_{2}$ in order to immobilize the metal on the support surface (Fig. 20). Acetylacetone substituted by a trimethoxysilyl group, 3-[3-(trimethoxysilyl)propyl]-2,4-pentanedione (MSacac-H), was prepared and condensed on the surface of a non porous silica, whereas nucleophilic substitution of chloride in chloromethyl groups by sodium acetylacetonate was carried out for the Merrifield resin (Fig. 20a). The supported complexes were then used to control the bulk polymerization of vinyl acetate initiated by $\mathrm{V}-70$ at $30^{\circ} \mathrm{C}$ (Fig. 20b). The 
Supported Cobalt Mediated Radical Polymerization (SCMRP) of VAc was effective and produced PVAc with relatively narrow molar mass distribution [125]. At the end of the reaction, removal of the PVAc chains from the surface-grafted cobalt complex was successfully achieved by addition of a nitroxide (2,2,6,6-tetramethylpiperidinoxy, TEMPO), followed by centrifugation and recovery of the support (Fig. 20b). The successful re-use of the supported cobalt complex was quite encouraging, although partial metal passivation after each cycle was observed.

Although CMRP is still far from industrial development, all these contributions emphasize the difficulties and futures challenges for this system and pave the way in that direction.

\subsection{Macromolecular Design}

Nowadays, the design of novel polymer materials with well-defined structures and specific properties, aiming at the development of a large range of applications, is becoming an essential aspect of polymer science. In this respect, the contribution of controlled radical polymerization is remarkable. A large range of polymers with well-defined molecular characteristics (i.e. length, chain-end, architecture, composition, functionality,...) have been made available by CRP under mild conditions [126-129]. In that context, the ability of CMRP to control the polymerization of difficult monomers constitutes a new opportunity in macromolecular engineering. The approaches that have so far been explored for producing functionalized polymers, copolymers and more complex architectures by CMRP are mentioned below.

\subsubsection{End-functionalization}


One major advantage of CRP relative to living ionic polymerizations is its tolerance towards many functional groups allowing the preparation of a large range of telechelic polymers without using protecting groups. Typical examples of end-functionality control for polymers prepared by CMRP are depicted in Fig. 21 .

The $\alpha$-end group is controlled by the initiator structure. Organocobaloximes containing various functional groups, such as phenyl, bromide, hydroxyl, carboxyl and nitrile groups, were prepared and used as photoinitiators to produce $\alpha$-functionalized poly(acrylates) (Fig. 21a) [65]. For the EA polymerization initiated by 6-bromohexyl (pyridinato)cobaloxime, NMR analyses indicated that about $90 \%$ of the PEA chains contained a bromine atom at the $\alpha$ chain end. At the end of the CMRP process, most of the chains are end-capped by the cobalt complex used as controlling agent. Therefore, the well known reactivity of alkylcobalt(III) complexes [130-132] can be used to convert and functionalize the polymer $\omega$ chain end. A few methods have already been successfully applied. For example, photolytic treatment of PEA-Co(DMG $)_{2}(\mathrm{Py})$ in the presence of bis( $p$-aminophenyl) disulfide yielded a polyacrylate having a terminal aminophenylthioether function (Fig. 21b) [65]. Well-defined polyacrylate macromonomers were obtained upon addition of methyl methacrylate on the same PEA$\mathrm{Co}(\mathrm{DMG})_{2}(\mathrm{Py})$ by subsequent radical addition and catalytic chain transfer reaction (Fig. 21c) [65]. PVAc-Co(acac) $)_{2}$ has also been quenched by various scavengers. Addition of alkanethiol to the polymerization medium terminates the chains by a proton and allowed the removal of the cobalt complex (Fig. 21d) [124]. Similarly, TEMPO but also functionalized nitroxides (with epoxy [124], $\alpha$-bromo ketone and $\alpha$-bromo ester [133] groups) scavenged the PVAc radicals released by the thermal $\mathrm{Co}-\mathrm{C}$ bond cleavage (Fig. 21e). Since the covalent bond between PVAc and nitroxide is strong [18], this approach is valid for the functionalization of the PVAc chains [124]. Indeed, it is reported that $N$-alkoxyamines with a heteroelement in the 
$\alpha$ position (O in the case of PVAc) present markedly stronger C-TEMPO bond [134]. After treatment with TEMPO or thiols followed by elution through a silica column, the cobalt content in the polymer was drastically reduced. Finally, addition of functionalized, non activated olefins such as 1,2-epoxy-5-hexene and 3-butene-1-ol inhibited the CMRP of VAc leading to the chain end capping by an epoxy and hydroxyl group, respectively (Fig. 21f). Following the same principle, fullerenes were successfully grafted with poly(vinyl acetate) and poly $(N$-vinyl pyrrolidone) (PNVP) chains by radical addition to the double bond of the $\mathrm{C}_{60}$ (Fig. 21g) [135, 136]. An average number of four chains were detected when a large excess of PVAc-Co(acac) 2 was reacted with $\mathrm{C}_{60}$ whereas mixtures of nanohybrids with different grafting level (from 1 to 4 polymer chains) were collected when the [PVAc$\mathrm{Co}] /\left[\mathrm{C}_{60}\right]$ molar ratio was decreased. The well-defined hydrosoluble and potentially biocompatible PNVP-C 60 and poly(vinyl alcohol)- $\mathrm{C}_{60}\left(\mathrm{PVOH}-\mathrm{C}_{60}\right)$, obtained by hydrolysis of the PVAc ester functions) proved able to generate singlet oxygen upon photolytic treatment, making them promising agents for photodynamic cancer therapy $[135,136]$. Interestingly, compounds containing several cobalt moieties were synthesized and used as multifunctional CMRP initiator. For example, 1,6-bis[(dipyridinato)cobaloxime $]$ hexane $\left(\mathrm{Py}\left(\mathrm{dmgH}_{2}\right)_{2} \mathrm{Co}-\right.$ $\left(\mathrm{CH}_{2}\right)_{6}-\mathrm{Co}\left(\mathrm{dmgH}_{2}\right)_{2} \mathrm{Py}, 45$ in Fig. 22) was used as difunctional photoinitiator for the ethyl acrylate polymerization in chloroform [16, 65]. The resulting PEA $\left(M_{n}=19000 \mathrm{~g} / \mathrm{mol}\right.$, $M_{w} / M_{n}=2.4$ ) presented a Co-C bond at each chain end, opening the way to the formation of triblocks copolymers. Under similar experimental conditions, photoinitiators containing three $\left(\mathrm{CH}_{3} \mathrm{CH}_{2} \mathrm{C}\left[\mathrm{CH}_{2} \mathrm{CO}_{2} \mathrm{C}\left(\mathrm{CH}_{3}\right) \mathrm{Co}(\mathrm{DMG})_{2} \mathrm{Py}\right]_{3}, \quad 46\right.$ in Fig. 22) and four $\left(\mathrm{C}_{\mathrm{CH}} \mathrm{CH}_{2} \mathrm{C}\left(\mathrm{CH}_{3}\right) \mathrm{Co}(\mathrm{DMG})_{2} \mathrm{Py}\right]_{4}, 47$ in Fig. 22) cobaloxime moieties were used to promote the synthesis of star-shaped polyacrylates with three and four arms, respectively [65]. In these cases, $\mathrm{M}_{\mathrm{n}}$ increased linearly with the monomer conversion but were lower than the expected values ( $\mathrm{f}=\mathrm{M}_{\mathrm{n} \text { th }} / \mathrm{M}_{\mathrm{n} \exp }=1.25$ ), possibly due to transfer reactions. Although it is reasonable to 
assume that all the arms grew uniformly, additional experiments are necessary to confirm this conclusion.

\subsubsection{Copolymerization}

In the era of nanotechnology, the design of well-defined block copolymers able to selfassemble in bulk or in solution into nanodomains is becoming an important aspect of polymer synthesis. In that respect, CMRP has already contributed to the preparation of valuable copolymers.

Wayland was first to demonstrate the ability of CMRP to provide block copolymers $[15,73,75,77]$. Well-defined PMA chains $\left(M_{n}=40600 \mathrm{~g} / \mathrm{mol}, \mathrm{M}_{\mathrm{w}} / \mathrm{M}_{\mathrm{n}}=1.22\right)$ end-capped by (TMP)Co (30 in Table 2) and purified from the unreacted monomer were treated with $n \mathrm{BuA}$ and benzene. Upon thermal activation at $60^{\circ} \mathrm{C}$, a PMA- $b$-PnBuA block copolymer $\left(\mathrm{M}_{\mathrm{n}}=\right.$ $131400 \mathrm{~g} / \mathrm{mol}, \mathrm{M}_{\mathrm{w}} / \mathrm{M}_{\mathrm{n}}=1.29$ ) was formed (Fig. 23a).[15] Although the controlled character of the CMRP was indisputably established, the range of accessible copolymers has long remained limited to acrylic monomers. In a similar approach, a PEA- $b-\mathrm{P} n \mathrm{BuA}$ block copolymer $(25800 \mathrm{~g} / \mathrm{mol})$ was prepared by cobaloxime-mediated radical polymerization by sequential polymerization of EA and $n \mathrm{BuA}$ upon photolytic treatment (Fig. 23c). The block sequencing occurred relatively efficiently provided that growth of the first PEA block (7400 $\mathrm{g} / \mathrm{mol}, \mathrm{M}_{\mathrm{w}} / \mathrm{M}_{\mathrm{n}}=1.6$ ) was stopped before reaching high conversions $(<60 \%)$ [65]. Finally, a $\mathrm{P} n \mathrm{BuA}-b$-PEA- $b-\mathrm{P} n \mathrm{BuA}$ triblock copolymer $\left(\mathrm{M}_{\mathrm{n}}=33500 \mathrm{~g} / \mathrm{mol}, \mathrm{M}_{\mathrm{w}} / \mathrm{M}_{\mathrm{n}}=2.7\right)$ was obtained by $n \mathrm{BuA}$ polymerization initiated by a difunctional PEA macroinitiator $\left(\mathrm{M}_{\mathrm{n}}=19000 \mathrm{~g} / \mathrm{mol}\right.$, $\mathrm{M}_{\mathrm{w}} / \mathrm{M}_{\mathrm{n}}=2.4$ ) obtained from 1,6-hexyl[(dipyridinato)cobaloxime] 45 (Fig. 23d) [65].

As the controlling ability of cobalt porphyrin and cobaloximes has long remained limited to acrylic monomers, the range of accessible block copolymers was restricted as well. 
An exception is the VAc polymerization initiated by a Co(TMP)-capped PMA $\left(\mathrm{M}_{\mathrm{n}}=30000\right.$ $\left.\mathrm{g} / \mathrm{mol}, \mathrm{M}_{\mathrm{w}} / \mathrm{M}_{\mathrm{n}}=1.11\right)$, which led to well-defined PMA- $b$-PVAc $\left(\mathrm{M}_{\mathrm{n}}=85000 \mathrm{~g} / \mathrm{mol}, \mathrm{M}_{\mathrm{w}} / \mathrm{M}_{\mathrm{n}}=\right.$ 1.21) at low monomer conversion (VAc conversion $=9 \%)($ Fig. 23b) $[101,102]$. On the other hand, the use of bis(acetylacetonato)cobalt(II) and derivatives significantly enlarged the range of copolymers made available by CMRP. The first efforts were directed to the statistical copolymerization of VAc with various monomers such as acrylate [95], NVP [137], and chlorovinyl acetate $[137,138]$. Although acrylates polymerized in a completely uncontrolled fashion in the presence of $\mathrm{Co}(\mathrm{acac})_{2}$, random copolymerization of $\mathrm{nBuA}$ with $\mathrm{VAc}$ followed a controlled pathway (Fig. 24a) [95]. According to the ${ }^{1} \mathrm{H}$ NMR study, nBuA was incorporated preferentially in the early stages of the copolymerization leading to a gradient sequence distribution in the $\mathrm{P}_{\mathrm{n}} \mathrm{BuA}-\mathrm{co}-\mathrm{PVAc}$ copolymer. Typically, polydispersities as low as 1.2-1.3 could be obtained provided that the initial feed had a high VAc content (above 50\%), indicating that deactivation of the VAc-terminated radical chain by the cobalt complex was the critical step to control the copolymerization.

A similar behavior was observed when the statistical copolymerization of NVP with VAc was initiated by V-70 in the presence of $\mathrm{Co}(\mathrm{acac})_{2} 39$ (Fig. 24b) [137]. The synthesis of PNVP-co-PVAc with low polydispersities was achieved in spite of the moderate control observed for the polymerization of NVP alone with the same complex [137]. VAc was also successfully copolymerized with vinyl chloroacetate (VClAc) by CMRP using $\operatorname{Co}\left(\mathrm{F}_{3} \mathrm{acac}\right)_{2} \mathbf{4 1}$ as controlling agent, leading to well-defined PVAc-co-PVClAc copolymers (Fig. 24c) [137, 138]. The pendant $\alpha$-chloro ester functions of this statistical copolymer were then used as initiators for the styrene atom transfer radical polymerization (ATRP) catalyzed by copper chloride and bis(2-pyridylmethyl)octadecylamine (BPMODA) providing the corresponding PVAc-graft-PS (Fig. 24c) [138]. Hydrolysis of the PVAc-graft-PS copolymer ester functions allowed releasing the PS side chains for size exclusion chromatography (SEC) analyses. The 
latter confirmed the monomodal molar mass distribution of the PS segments in spite of a slight tailing toward the low molar mass region.

Since the CMRP method based on $\mathrm{Co}(\mathrm{acac})_{2}$ was not able to impart control to monomers such as styrene, acrylates and methacrylates, the straightforward synthesis of welldefined PVAc-containing block copolymers by CMRP alone appeared difficult. Therefore, CMRP was combined with ATRP to tackle this problem (Fig. 25). Typically, PVAc$\mathrm{Co}(\mathrm{acac})_{2}$ chains preformed by CMRP were end-functionalized by nitroxides containing $\alpha$ bromo ketone or $\alpha$-bromo ester [133]. The activated halogen was then used to initiate the ATRP of styrene, methyl methacrylate and ethyl acrylate to provide the corresponding welldefined block copolymers [133]. This appeared as a valuable approach to yield thermally robust copolymers, because the nitroxide-PVAc bond is known to be strong [18]. Indeed, SEC analysis of the PVAc- $b$-PMMA copolymer before and after thermal treatment at $180^{\circ} \mathrm{C}$ remained unchanged [133].

Although efficient, this three-step approach towards block copolymers is time consuming and the real challenge remained to carry out block copolymerization by CMRP only. Therefore, in spite of the poor control reported for the CMRP of styrene, the initiation of this monomer was realized from a PVAc-Co(acac $)_{2}$ macroinitiator upon thermal treatment (Fig. 26a). As expected, a PVAc- $b$-PS copolymer with a well-defined PVAc block and an illdefined PS segment was obtained. Methanolysis of the PVAc block led to an amphiphilic PVOH- $b$-PS copolymer that was shown to self-assemble into nanocups (bowl-shaped vesicles) in water [139].

Similarly, the synthesis of PVAc- $b$-polyalkene copolymers was attempted by initiation of ethylene and 1 -octene at $30^{\circ} \mathrm{C}$ from a well-defined PVAc-Co(acac $)_{2}$ macroinitiator. However, although the initiation of the polyalkene segment occurred, the monomer conversion was limited to about $15 \%$ and the length of this second block was also quite 
limited (DP 4) due to the low reactivity of these non activated monomers (Fig. 26b) [140]. Increasing the polymerization temperature to $60^{\circ} \mathrm{C}$ did not allow circumventing this problem. Methanolysis of the PVAc block of these products yielded amphiphilic poly(vinyl alcohol)- $b$ poly(1-octene). When the copolymerization of vinyl acetate with 1-octene was initiated by V70 in the presence of $\mathrm{Co}(\mathrm{acac})_{2}$ or by a poly(vinyl acetate) chain end-capped by $\mathrm{Co}(\mathrm{acac})_{2}$, vinyl acetate was preferentially incorporated, such that a copolymer with a gradient structure was formed.

The same strategy was also applied to synthesize well-defined PVAc- $b$-PNVP by CMRP (Fig. 26c) [141]. The optimal conditions established for the polymerization of NVP from the $\mathrm{Co}(\mathrm{acac})_{2}$-terminated $\mathrm{PVAc}$ were $30^{\circ} \mathrm{C}$ in anisole. Under these conditions, all criteria of a CRP process were met and copolymers with a polydispersity around 1.3 were formed. The amphiphilic PVAc- $b$-PNVP copolymers were then easily transformed into the hydrosoluble and potentially biocompatible PVOH- $b$-PNVP block copolymers [141].

Finally, the same approach was applied to the synthesis of PVAc- $b$-PAN block copolymers and the solvent choice turned out to be crucial [142]. Indeed, when carried out at $0^{\circ} \mathrm{C}$ in anisole, a slow and incomplete initiation of acrylonitrile from the PVAc-Co(acac $)_{2}$ chains was observed leading to block copolymer contaminated by unreacted PVAc. However, at the same temperature, all the PVAc-Co(acac $)_{2}$ chains initiated simultaneously the acrylonitrile polymerization when using dimethylformamide as solvent, allowing the formation of well-defined PVAc- $b$-PAN (Mw/Mn 1.1) (Fig. 26d) [142].

The DFT analysis provides a rationalization of this phenomenon. As shown in Fig. 27 , the dormant species for the (acac) ${ }_{2} \mathrm{Co}^{\mathrm{III}}-\mathrm{PVAc}$ contains a 6 -coordinate complex with a chelated $\left(\kappa^{2}: O, C\right)$ ester function as chain end in a non-coordinating solvent. AN coordination does not provide sufficient stabilization to open this chelate, contrary to DMF, whereas DMSO provides only weak stabilization. In the absence of solvent, the (acac) ${ }_{2} \mathrm{Co}-\mathrm{PVAc}$ 
dormant chain must dissociate to tetrahedral $\mathrm{Co}(\mathrm{acac})_{2}$, which costs $13.1 \mathrm{kcal} \mathrm{mol}^{-1}$ in enthalpy, because AN is not a sufficiently strong ligand to coordinate. After conversion of the PVAc radical to a PAN radical by AN addition, subsequent trapping yields the (acac) ${ }_{2} \mathrm{Co}^{\mathrm{III}}$ PAN dormant chain, regaining $6.7 \mathrm{kcal} \mathrm{mol}^{-1}$. This dormant chain is not strongly coordinated by $\mathrm{AN}$, since this process is entropically disfavoured as discussed in a previous section. The comparison of the enthalpies involved $\left(13.1 \mathrm{kcal} \mathrm{mol}^{-1} \text { for the activation of (acac) }\right)_{2} \mathrm{Co}-\mathrm{PVAc}$ vs. $6.7 \mathrm{kcal} \mathrm{mol}^{-1}$ for the reactivation of (acac) $\left.{ }_{2} \mathrm{Co}-\mathrm{PAN}\right)$ shows that crossing over from a PVAc block to a PAN block yields slow initiation in a non-coordinating solvent, in agreement with the experimental observation. In DMSO or in DMF, on the other hand, the activation of the $(\mathrm{L})(\mathrm{acac})_{2} \mathrm{Co}-\mathrm{PVAc}$ dormant chain (yielding in these cases $\left.\mathrm{Co}(\mathrm{acac})_{2}(\mathrm{~L})_{2}\right)$ requires an enthalpy only marginally greater than the reactivation of the subsequent (L)(acac) ${ }_{2} \mathrm{Co}-\mathrm{PAN}$ dormant chain. In DMF, the radical activation equilibrium has an enthalpy of $10.8 \mathrm{kcal} \mathrm{mol}^{-1}$ for the Co-capped PVAc vs. $9.2 \mathrm{kcal} \mathrm{mol}^{-1}$ for the Co-capped PAN, whereas in DMSO these values are 7.1 and $6.6 \mathrm{kcal} \mathrm{mol}^{-1}$, respectively. Thus, crossing over from a PVAc block to a PAN block is more efficient in the coordinating solvents, particularly DMSO where these two enthalpies are more similar. The main reason for this difference is that coordinating solvents are able to compete with the internal stabilization provided by the chelating ester function for the PVAc dormant species, whereas the PAN dormant chain has no mechanism available for internal stabilization. It can also be observed that the activation is energetically less costly in DMSO, leading to faster polymerization, which agrees with the experimental evidence.

It was also demonstrated that these PVAc- $b$-PAN copolymers are interesting precursors for novel PVOH-containing copolymers such as hydrosoluble PVOH- $b$-PAA [142] and amphiphilic PVOH- $b$-PAN [143]. The former resulted from hydrolysis of both the ester and the nitrile functions with potassium hydroxide in ethanol/water at $75^{\circ} \mathrm{C}$ (Fig. 26e) whereas the latter was obtained by selective hydrolysis of the ester function of the parent 
PVAc containing copolymer (Fig. 26d). The pH responsiveness of the PVOH- $b$-PAA copolymer was demonstrated by Dynamic Light Scattering pH titration [142]. This copolymer also proved ability to form spherical micelles in aqueous solutions with an oppositely charged double hydrophilic diblock copolymer (poly(2-methylvinylpyridinium iodide)-blockpoly(ethylene oxide), P2MVP- $b$-PEO) [144]. Finally, the PVOH- $b$-PAN copolymer was used as a template for the preparation of gold loaded carbon nano-objects. [143]

\section{Concluding remarks and perspectives}

Considerable progress has been made in the last decade in the field of controlled radical polymerization (CRP) and the design of well-defined polymer architectures. Among several CRP techniques, Cobalt Mediated Radical Polymerization (CMRP) distinguished itself by its ability to mediate the polymerization of monomers of very different reactivity such as vinyl acetate and acrylates. This specificity justifies that efforts are currently devoted to the improvement of this CRP method and those based on metallic species in general.

This review condenses and comments all the work done in CMRP since its discovery sixteen years ago. In summary, it allows a controlled radical polymerization of acrylic esters, acrylic acid, vinyl esters, acrylonitrile and vinylpyrrolidone, leading to the corresponding well-defined polymers with predictable molar masses. One interesting characteristic is the possibility to target high molecular weights (above $200000 \mathrm{~g} / \mathrm{mol}$ in some cases) with good control at high conversions. Another striking specificity is the low polymerization temperature, ranging from $60^{\circ} \mathrm{C}$ in the case of acrylates mediated by cobalt porphyrins, to $0^{\circ} \mathrm{C}$ for acrylonitrile in the presence of $\mathrm{Co}(\mathrm{acac})_{2}$.

From the mechanistic point of view, the CMRP process has long been considered to occur only by reversible deactivation of the growing chains by the metal, whatever the nature 
of the cobalt complex and the experimental conditions. More recently, the contribution of the degenerative chain transfer mechanism has been emphasized. Mechanistic studies have also highlighted the crucial role of additives, especially in the case of $\operatorname{Co}(\mathrm{acac})_{2}$. Indeed, addition of Lewis bases such as water, pyridine, dimethylsulfoxide and dimethylformamide, capable of coordinating the cobalt center, deeply modifies the chain activation equilibrium and drives the process towards the reversible termination mechanism.

To date, the main classes of cobalt complexes that have shown activity in CMRP are cobaloximes, cobalt porphyrins and $\beta$-diketonato derivatives of cobalt(II). The first two families are particularly efficient mediators for the acrylic esters and acrylic acid polymerizations whereas the last category has so far been found to control the polymerization of vinyl esters, acrylonitrile and vinylpyrrolidone. These observations emphasize that the success of CMRP largely depends on the choice of the monomer/cobalt complex couple.

As it stands today, the field still faces many challenges. First, it is crucial to enlarge the synthetic possibilities of the CMRP and to fine tune both the experimental conditions and the cobalt complex structure in order to control more monomers such as styrenic derivatives, isoprene, etc... In the quest for the adequate cobalt complex/monomer couple or for a controlling agent as universal as possible, computational chemistry might be used as a predicting tool. This is all the more realistic since DFT calculations have already proved the ability to rationalize experimental observations based on the evaluation of the $\mathrm{Co}-\mathrm{C}$ bond dissociation enthalpy (BDE) and the relative stabilization ability of several neutral donor molecules for the cobalt(II) "persistent radical". In future endeavours, the kinetic parameters, especially those related to the radical trapping and $\mathrm{H}$-transfer processes, must be investigated and combined to the already available thermodynamic data in order to approach the system as a whole. The future implementation of CMRP to other monomers should considerably enlarge the macromolecular engineering possibilities. The potential to synthesize novel block 
copolymers and more complex architectures has been highlighted in this review. The main difficulty consists in finding a cobalt complex able to control different co-monomers in order to obtain copolymers with well-defined sequences. A valuable approach, already proven successful for the synthesis of well-defined PVAc- $b$-PAN copolymers, consists of tuning the cobalt reactivity on going from one block to the next one upon introduction of additives with coordinating power. This innovative approach clearly highlights the versatility of CMRP and evidences its potential for accessing new copolymers.

The second challenge consists of bringing the technique closer to industrial standards. As described in this review, a few approaches have already been envisioned. For example, the CMRP has been successfully applied in aqueous dispersed media such as suspensions and miniemulsions. However, questions relative to the recovery and the recycling of the cobalt complex, used in large amounts in the polymerization, remain pending and need to be addressed. Immobilization of the cobalt complex on an inert support has already been tested with encouraging results. Such an approach certainly deserves further efforts to improve the process efficiency and to preserve the complex activity through multiple recycles.

\section{Acknowledgements}

The authors from Liège are indebted to the "Belgian Science Policy" for financial support in the frame of the "Interuniversity Attraction Poles Programme (PAI VI/27) Functional Supramolecular Systems", and to the "Fonds National de la Recherche Scientifique" (F.N.R.S., Belgium). A. D. (“Chargé de Recherches FNRS”) and C. D. ("Maître de Recherches FNRS") thank F.N.R.S for funding. R.P. thanks the Institut Universitaire de France and the "Agence National de la Recherche" (Contract No. NT05-2_42140) for 
funding, and the "Centre Interuniversitaire de Calcul de Toulouse" (Project CALMIP) for granting free computational time.

\section{References}

As suggested, the references are in a table in a separate file.

\section{Figure Captions}

Fig. 1. Photolytic transformation of methylcobalamine into acetylcobalamin.

Fig. 2. Thermal isomerization of coenzyme B12 model.

Fig. 3. Cobalt-mediated vinylation involving alkylcobaloximes or acylcobalt salophen reagents.

Fig. 4. General mechanism of carbon-carbon bond formation by reaction of organocobalt complexes with olefins.

Fig. 5. Examples of cobalt complexes active in catalytic chain transfer polymerization (CCTP).

Fig. 6. Interplay of hydrogen transfer reactions and persistent radical effect (PRE) for polymerizations conducted in the presence of cobalt complexes.

Fig. 7. General mechanism of cobalt mediated radical polymerization.

Fig. 8. Initiation mechanism of the cobalt porphyrin-mediated radical polymerization.

Fig. 9. Equilibrium between dormant and active species in the CMRP of vinyl acetate using bis(acetylacetonato)cobalt(II) $\mathbf{3 9}$ as controlling agent.

Fig. 10. Initiation mechanism of the $\mathrm{Co}(\mathrm{acac})_{2}$-mediated radical polymerization of vinyl acetate.

Fig. 11. Mechanism of the CMRP of VAc in the presence of Lewis bases (e.g. pyridine). 
Fig. 12. Interplay of degenerative chain transfer (A) and reversible termination (B) mechanisms in the CMRP of VAc.

Fig. 13. CMRP of acrylonitrile initiated by a preformed alkylcobalt(III) adduct (44) in dimethylsulfoxide.

Fig. 14. Qualitative diagram relating the enthalpy of the $\mathrm{Co}^{\mathrm{III}}$ and $\mathrm{Co}^{\mathrm{II}}$ complexes implicated in CMRP.

Fig. 15. Qualitative energy diagram for the $\mathrm{Co}^{\mathrm{III}}-\mathrm{R}$ homolytic bond rupture: (a) porphyrin and corrin systems, giving low spin $(\mathrm{S}=1 / 2) \mathrm{Co}^{\mathrm{II}}$ complexes; (b) bis(acetylacetonato) system, giving high spin $(\mathrm{S}=3 / 2)$ complexes.

Fig. 16. Energy diagram obtained with the B3PW91* functional for the Co-C homolytic bond cleavage and subsequent $\mathrm{L}$ addition in $(\mathrm{acac})_{2}(\mathrm{~L}) \mathrm{Co}-\mathrm{CH}(\mathrm{CN}) \mathrm{CH}_{3}(\mathrm{~L}=$ nothing, AN, DMSO). The values shown are relative enthalpies in $\mathrm{kcal} \mathrm{mol}^{-1}$.

Fig. 17. Qualitative reaction coordinates for the reversible activation of an organometallic dormant chain. Solid curves correspond to $S=0$ and the dashed curve to $S=1$.

Fig. 18. Qualitative reaction coordinate for the associative radical exchange on an organometallic dormant chain with low (e) and high (d) energy barrier.

Fig. 19. Schematic representation of the VAc CMRP in aqueous dispersed media (suspension and miniemulsion); acceleration of the polymerization in the monomer phase by diffusion of the cobalt(II) deactivating species into the water phase.

Fig. 20. General strategy for supported cobalt-mediated radical polymerization (SCMRP) : (a) $\mathrm{Co}(\mathrm{acac})_{2}$ immobilization onto silica or Merrifield resin ; (b) polymerization involving the supported cobalt(II) complex followed by metal recovery and polymer purification.

Fig. 21. End-functionalization of the polymer chains in CMRP.

Fig. 22. CMRP photoinitiators containing several cobaloximes moieties. 
Fig. 23. Synthesis of block copolymers by CMRP.

Fig. 24. Synthesis of linear and graft copolymers by CMRP.

Fig. 25. Synthesis of PVAc containing block copolymers by combination of CMRP and ATRP.

Fig. 26. Synthesis of PVAc and PVOH containing block copolymers by CMRP.

Fig. 27. Energy diagram obtained with the B3PW91* functional for the Co-C homolytic bond cleavage in $(\mathrm{acac})_{2} \mathrm{Co}-\mathrm{CH}\left(\mathrm{CH}_{3}\right)\left(\mathrm{OCOCH}_{3}\right)$ and subsequent trapping of the $\mathrm{CH}(\mathrm{CN}) \mathrm{CH}_{3}$ radical in the presence of different neutral donors $(\mathrm{L}=\mathrm{AN}, \mathrm{DMF}$, DMSO). The values shown are relative enthalpies in $\mathrm{kcal} \mathrm{mol}^{-1}$. 
Table 1

Cobaloximes derivatives.

\begin{tabular}{llllll}
\hline Structure & $\mathbf{X}$ & $\mathbf{Y}$ & $\mathbf{R}$ & Name & $\mathbf{N}^{\circ}$ \\
\hline & $-\mathrm{H}$ & $/$ & $/$ & $\mathrm{Co}(\mathrm{dmgH})_{2}$ & $\mathbf{1 3}$ \\
\hline
\end{tabular}


Table 2

Cobalt porphyrin derivatives.

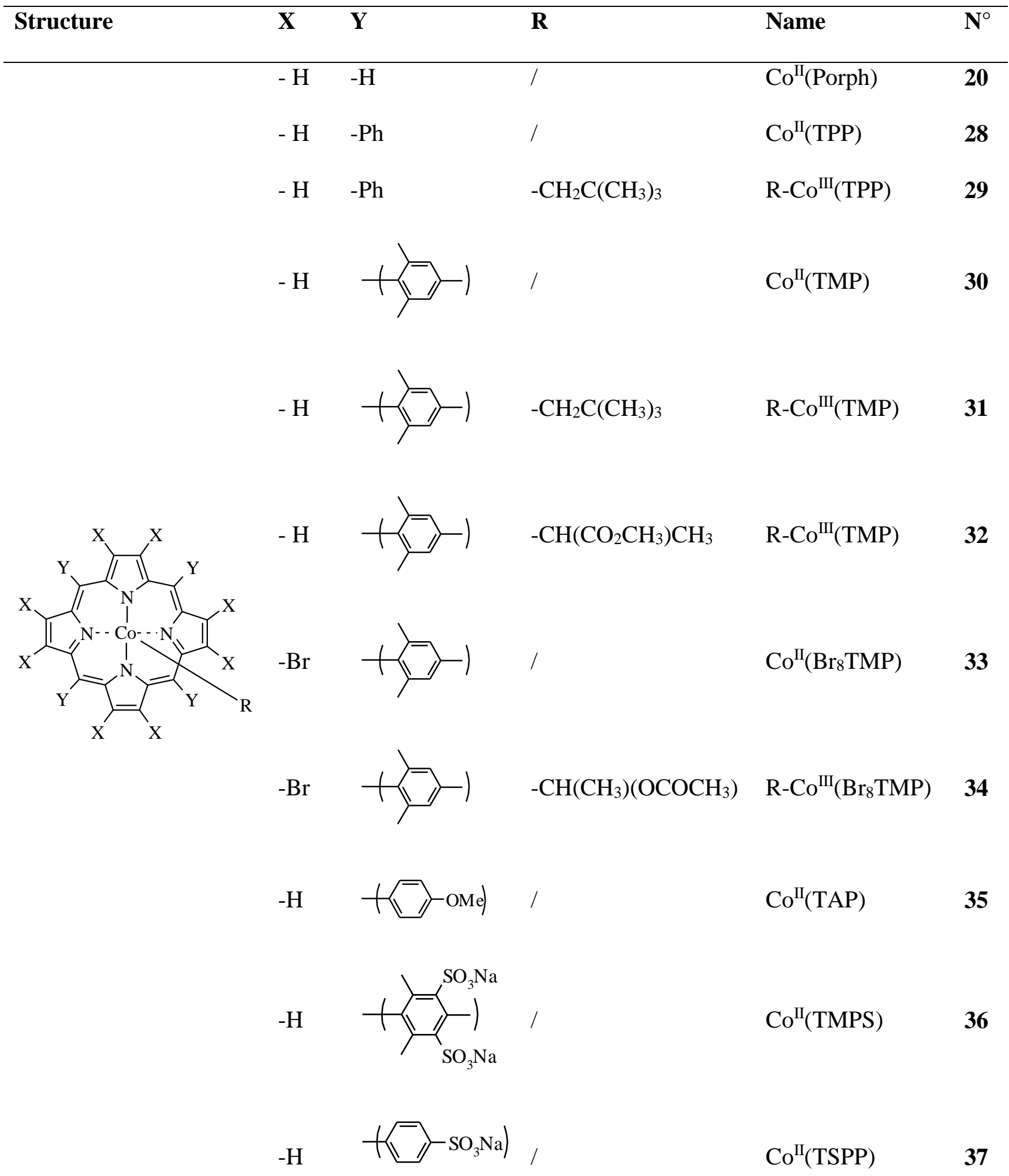




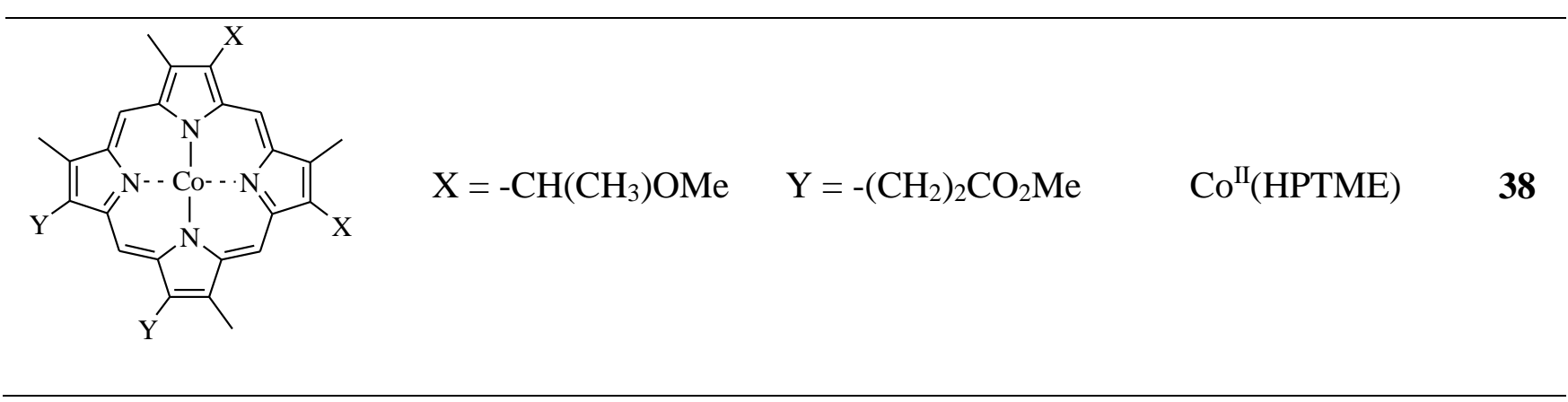




\section{Table 3.}

Bis( $\beta$-diketonato)cobalt(II) derivatives and Bis( $\beta$-diketonato)cobalt(III) compounds used in CMRP.

\begin{tabular}{llllll}
\hline Cobalt(II) & X & Y & R & Name & $\mathbf{N}^{\circ}$
\end{tabular}
complex

\begin{tabular}{lllll}
\hline & $-\mathrm{CH}_{3}$ & $/$ & $\mathrm{Co}(\mathrm{acac})_{2}$ & 39 \\
$-\mathrm{C}\left(\mathrm{CH}_{3}\right)_{3}$ & $-\mathrm{C}\left(\mathrm{CH}_{3}\right)_{3}$ & $/$ & $\mathrm{Co}(\mathrm{TMHD})_{2}$ & $\mathbf{4 0}$ \\
$-\mathrm{CF}_{3}$ & $-\mathrm{CH}_{3}$ & $/$ & $\mathrm{Co}\left(\mathrm{F}_{3}-\mathrm{acac}\right)_{2}$ & $\mathbf{4 1}$ \\
$-\mathrm{CF}_{3}$ & $-\mathrm{CF}_{3}$ & $/$ & $\mathrm{Co}\left(\mathrm{F}_{6}-\mathrm{acac}\right)_{2}$ & $\mathbf{4 2}$ \\
& $-\mathrm{CH}_{3}$ & $-\mathrm{CH}(\mathrm{OAc}) \mathrm{CH}_{3}$ & $\mathrm{R}-\mathrm{Co}(\mathrm{acac})_{2}$ & $\mathbf{4 3}$ \\
& $-\mathrm{CH}_{3}$ & $-\left(\mathrm{CH}(\mathrm{OAc}) \mathrm{CH}_{2}\right)_{44}-\mathrm{R}_{0}{ }^{*}$ & $\mathrm{R}-\mathrm{Co}(\mathrm{acac})_{2}$ & $\mathbf{4 4}$
\end{tabular}

$* \mathrm{R}_{0}$ being an $\mathrm{V}-70$ initiating fragment : $-\mathrm{CH}(\mathrm{CN})\left(\mathrm{CH}_{3}\right)-\mathrm{CH}_{2}-\mathrm{C}\left(\mathrm{OCH}_{3}\right)\left(\mathrm{CH}_{3}\right)_{2}$. 
Table 4.

DFT-calculated bond dissociation enthalpies (BDE) at $298 \mathrm{~K}$ for R-Co ${ }^{\mathrm{III}}(\mathrm{acac})_{2}(\mathrm{~L})$ complexes. $^{\mathrm{a}}$

BDE (kcal mol-1)

\begin{tabular}{|c|c|c|c|c|}
\hline $\mathbf{R}$ & $\mathbf{L}$ & B3LYP & B3PW91* & Ref. \\
\hline $\mathrm{CH}_{3}$ & - & 14.6 & & [94] \\
\hline $\mathrm{CH}_{3}$ & py & 17.9 & & [94] \\
\hline $\mathrm{CH}_{3}$ & $\mathrm{NMe}_{3}$ & 17.2 & & [94] \\
\hline $\mathrm{CH}_{3}$ & $\mathrm{NH}_{3}$ & 17.8 & & [94] \\
\hline $\mathrm{CH}_{3}$ & $\mathrm{H}_{2} \mathrm{O}$ & 18.8 & & [94] \\
\hline $\mathrm{CH}\left(\mathrm{OCH}_{3}\right) \mathrm{CH}_{3}$ & - & 8.4 & & [99] \\
\hline $\mathrm{CH}\left(\mathrm{OOCCH}_{3}\right) \mathrm{CH}_{3}$ & - & 5.7 & 10.0 & {$[99,104]$} \\
\hline $\mathrm{CH}\left(\mathrm{OOCCH}_{3}\right) \mathrm{CH}_{3}$ & AN & 10.1 & 16.2 & [104] \\
\hline $\mathrm{CH}\left(\mathrm{OOCCH}_{3}\right) \mathrm{CH}_{3}$ & $\mathrm{DMF}$ & 8.2 & 14.1 & [104] \\
\hline $\mathrm{CH}\left(\mathrm{OOCCH}_{3}\right) \mathrm{CH}_{3}$ & DMSO & 4.5 & 9.9 & [104] \\
\hline $\mathrm{CH}\left(\mathrm{OOCCH}_{3}\right) \mathrm{CH}_{3}-\left(\kappa^{2}: C, O\right)$ & - & 11.9 & 13.0 & [99] [104] \\
\hline $\mathrm{CH}(\mathrm{CN}) \mathrm{CH}_{3}$ & - & 1.3 & 6.7 & [104] \\
\hline $\mathrm{CH}(\mathrm{CN}) \mathrm{CH}_{3}$ & AN & 7.7 & 14.2 & [104] \\
\hline $\mathrm{CH}(\mathrm{CN}) \mathrm{CH}_{3}$ & $\mathrm{DMF}$ & 6.0 & 12.5 & [104] \\
\hline $\mathrm{CH}(\mathrm{CN}) \mathrm{CH}_{3}$ & DMSO & 2.9 & 9.4 & [104] \\
\hline $\mathrm{CH}\left(\mathrm{COOCH}_{3}\right) \mathrm{CH}_{3}$ & - & -1.5 & & [99] \\
\hline $\mathrm{CH}\left(\mathrm{CH}_{3}\right)_{2} \mathrm{CN}$ & - & -5.5 & & [99] \\
\hline
\end{tabular}

${ }^{\mathrm{a}}$ Only bond energies were given for the bonds reported in ref. [94]. The values reported here are the standard enthalpies, obtained by corrections with the PV term at $298 \mathrm{~K}$. ${ }^{b} \mathrm{With}$ both 
functional, the calculations were carried out using the $6-31 \mathrm{G}^{* *}$ basis set for the light atoms $(\mathrm{C}, \mathrm{N}, \mathrm{O}, \mathrm{H})$ and the LANL2DZ basis set with an added $\mathrm{f}$ polarization function for Co. 
Table 5.

DFT-calculated Co-L bond dissociation enthalpies (BDE) at $298 \mathrm{~K}$ for $\mathrm{RCo}^{\mathrm{III}}(\mathrm{acac})_{2}(\mathrm{~L})$, $\mathrm{Co}^{\mathrm{II}}(\mathrm{acac})_{2}(\mathrm{~L})$ and $\mathrm{Co}^{\mathrm{II}}(\mathrm{acac})_{2}(\mathrm{~L})_{2}$ complexes. $^{\mathrm{a}}$

BDE (kcal mol-1) $)^{\mathbf{b}}$

\section{B3LYP B3PW91* Ref.}

$\operatorname{RCo}^{I I I}(\operatorname{acac})_{2}(\mathrm{~L})$ complexes

$\mathbf{R}$

$\mathbf{L}$

$\mathrm{CH}_{3}$

$\mathrm{H}_{2} \mathrm{O}$

9.9

[94]

$\mathrm{CH}_{3}$

$\mathrm{NH}_{3}$

11.7

[94]

$\mathrm{CH}_{3}$

$\mathrm{NMe}_{3}$

6.2

$\mathrm{CH}_{3}$

Py

7.7

[94]

$\mathrm{CH}\left(\mathrm{OOCCH}_{3}\right) \mathrm{CH}_{3}$

AN

$-0.23$

0.7

[104]

$\mathrm{CH}\left(\mathrm{OOCCH}_{3}\right) \mathrm{CH}_{3}$

DMF

6.8

7.0

[104]

$\mathrm{CH}\left(\mathrm{OOCCH}_{3}\right) \mathrm{CH}_{3}$

DMSO

4.4

3.8

[104]

$\mathrm{CH}(\mathrm{CN}) \mathrm{CH}_{3}$

AN

1.8

1.9

[104]

$\mathrm{CH}(\mathrm{CN}) \mathrm{CH}_{3}$

DMF

9.1

8.7

[104]

$\mathrm{CH}(\mathrm{CN}) \mathrm{CH}_{3}$

DMSO

7.5

6.6

[104]

$\operatorname{Co}^{I I}(\operatorname{acac})_{2}(L)$ complexes: $\mathrm{L}=$

AN

$\mathrm{CH}_{3} \mathrm{COOCH}_{3}$

$\mathrm{NMe}_{3}$

py

DMF

DMSO

$\mathrm{H}_{2} \mathrm{O}$
$-4.6$

$-5.6$

[104]

$-0.3$

[94]

3.5

[94]

4.3

[94]

4.3

2.9

[104]

5.9

3.9

[104]

5.8 
$\operatorname{Co}^{I I}(\operatorname{acac})_{2}(L)_{2}$ complexes: $\mathrm{L}=$
AN
1.2
2.4
[104]
$\mathrm{NMe}_{3}$
3.9
[94]
py
4.4
[94]
DMF
4.7
3.3
[104]
DMSO
4.6
2.8
[104]
$\mathrm{NH}_{3}$
12.0

${ }^{\mathrm{a}}$ Only bond energies were given for the bonds reported in ref. [94]. The values reported here are the standard enthalpies, obtained by corrections with the PV term at $298 \mathrm{~K}$. ${ }^{b} \mathrm{With}$ both functional, the calculations were carried out using the $6-31 G^{* *}$ basis set for the light atoms $(\mathrm{C}, \mathrm{N}, \mathrm{O}, \mathrm{H})$ and the LANL2DZ basis set with an added $\mathrm{f}$ polarization function for Co. 